\title{
PINK1 deficiency impairs mitochondrial homeostasis and promotes lung fibrosis
}

\author{
Marta Bueno, ${ }^{1,2}$ Yen-Chun Lai, ${ }^{1}$ Yair Romero, ${ }^{3}$ Judith Brands, ${ }^{1,2}$ Claudette M. St. Croix, ${ }^{4}$ Christelle Kamga, ${ }^{1}$ Catherine Corey, ${ }^{1}$ \\ Jose D. Herazo-Maya, ${ }^{5}$ John Sembrat, ${ }^{1,2}$ Janet S. Lee, ${ }^{2}$ Steve R. Duncan, ${ }^{2}$ Mauricio Rojas, ${ }^{2,6}$ Sruti Shiva,,${ }^{1,7}$ \\ Charleen T. Chu, ${ }^{8}$ and Ana L. Mora ${ }^{1,2}$ \\ 'Vascular Medicine Institute and '2Division of Pulmonary, Allergy and Critical Care Medicine, University of Pittsburgh, Pittsburgh, Pennsylvania, USA. ${ }^{3}$ Faculty of Sciences, Universidad Nacional Autónoma \\ de México, Ciudad Universitaria, Mexico City, Mexico. ${ }^{4}$ Center for Biological Imaging, University of Pittsburgh, Pittsburgh, Pennsylvania, USA. ${ }^{5}$ Pulmonary, Critical Care and Sleep Medicine, \\ Yale School of Medicine, New Haven, Connecticut, USA. ${ }^{6}$ Dorothy P. and Richard P. Simmons Center for Interstitial Lung Disease, 'Department of Pharmacology and Chemical Biology, and \\ ${ }^{8}$ Department of Pathology, University of Pittsburgh, Pittsburgh, Pennsylvania, USA.
}

\begin{abstract}
Although aging is a known risk factor for idiopathic pulmonary fibrosis (IPF), the pathogenic mechanisms that underlie the effects of advancing age remain largely unexplained. Some age-related neurodegenerative diseases have an etiology that is related to mitochondrial dysfunction. Here, we found that alveolar type II cells (AECIIs) in the lungs of IPF patients exhibit marked accumulation of dysmorphic and dysfunctional mitochondria. These mitochondrial abnormalities in AECIIs of IPF lungs were associated with upregulation of ER stress markers and were recapitulated in normal mice with advancing age in response to stimulation of ER stress. We found that impaired mitochondria in IPF and aging lungs were associated with low expression of PTEN-induced putative kinase 1 (PINK1). Knockdown of PINK1 expression in lung epithelial cells resulted in mitochondria depolarization and expression of profibrotic factors. Moreover, young PINK1-deficient mice developed similarly dysmorphic, dysfunctional mitochondria in the AECIls and were vulnerable to apoptosis and development of lung fibrosis. Our data indicate that PINK1 deficiency results in swollen, dysfunctional mitochondria and defective mitophagy, and promotes fibrosis in the aging lung.
\end{abstract}

\section{Introduction}

Age is a significant risk factor for the development of idiopathic pulmonary fibrosis (IPF): people older than 75 years have 50 times higher prevalence than people 35 years old or younger (1-3). The median age of diagnosis of IPF is approximately 66 years $(4,5)$. Whereas the familial disease has been related to mutations in surfactant proteins and telomerase, the cause of the majority of sporadic IPF cases and the mechanisms involved in aging-related IPF susceptibility are still unknown $(6,7)$. One of the most accepted theories in the pathogenesis of IPF is the vulnerability of type II alveolar epithelial cells (AECIIs) to endoplasmic reticulum (ER) stress, leading to "frustrated" re-epithelization, which is associated with secretion of profibrotic cytokines and fibroblast activation (8-10). The susceptibility to ER stress increases with age, providing a link to the central role of age as a risk factor for disease (11).

Mitochondria are particularly susceptible to age. Abnormalities in mitochondria are often observed with aging, including enlargement, loss of cristae, destruction of inner membranes, swelling, and impaired respiration (12). Interestingly, AECIIs in the lung are active progenitor and secretory cells with high energy demands, containing approximately $50 \%$ of lung mitochondrial mass $(13,14)$. Although mitochondrial structure and function are understood to be regulated by ER stress and autophagic processes

Conflict of interest: The authors have declared that no conflict of interest exists. Submitted: December 26, 2013; Accepted: November 13, 2014.

Reference information: J Clin Invest. 2015;125(2):521-538. doi:10.1172/JCI74942.
$(15,16)$, to our knowledge, there have been no studies to date evaluating mitochondria in AECIIs in human IPF or in mouse models of IPF. Our current investigations were informed by the central role of disordered PTEN-induced putative kinase 1 (PINK1) and mitochondrial dysfunction in age-related neurodegenerative diseases, and by our primary and unexpected observations of the numerous and large dysmorphic mitochondria evident on electron microscopic imaging of AECIIs from the lungs of patients with advanced IPF.

PINK1 is thought to have an important role in the maintenance of mitochondrial morphology and function and in the selective degradation of damaged mitochondria by mitophagy (17-19). PINK1 gene mutations are associated with an earlyonset familial form of Parkinson's disease (20), and altered regulation of the PINK1 locus has been found in patients with type 2 diabetes (21). PINK1 deficiency models in Drosophila and zebrafish, together with in vitro knockdown expression of PINK1, have demonstrated a significant reduction of electron transport chain (ETC) function by diminished ETC complex I and IV enzymatic activity, defective mitochondrial dynamics, increased oxidative stress, and altered mitophagy, leading cells to apoptosis (22-25). PINK1 is ubiquitously expressed (26), but PINK1 mutations can affect mitochondrial quality control differently according to cell type, age, and stress levels, which suggests that metabolic capacity and adaptation determine the cellular vulnerability to PINK1 deficiency in cells and animals (27). Our present studies showed that aging lungs are highly susceptible to ER stress, a key process that 
A
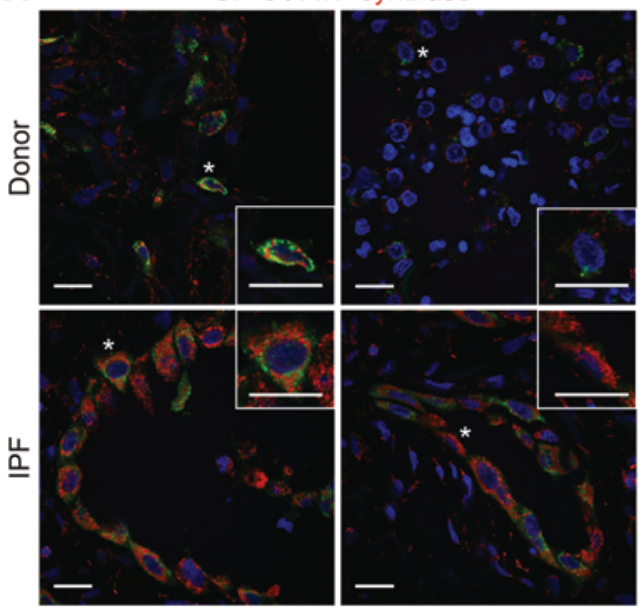

$\mathbf{F}$

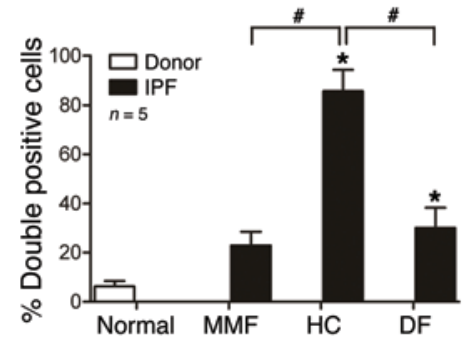

G

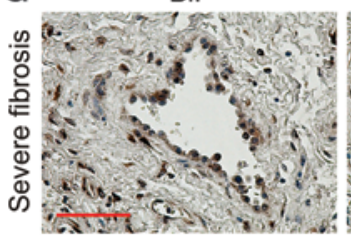

BiP

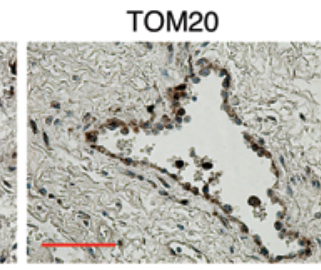

TOM20

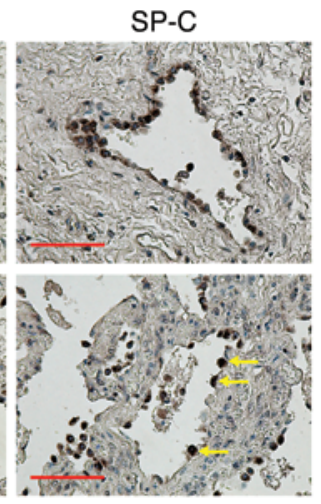

SP-C
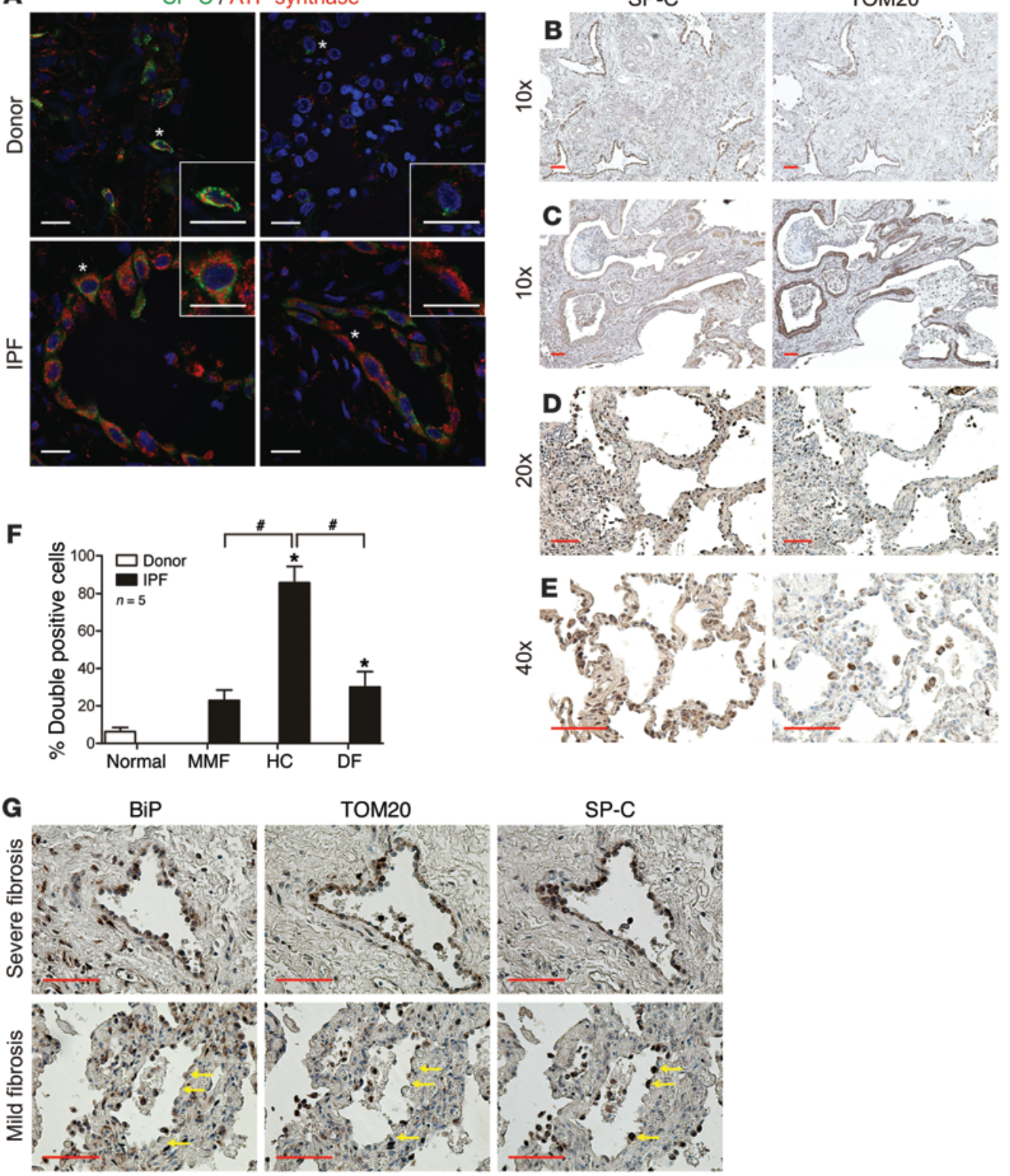

axishos triggers PINK1 downregulation and downstream effects including mitochondrial dysfunction, increased apoptosis, and activation of profibrotic responses.

\section{Results}

AECIIs from IPF lungs show accumulation of dysfunctional mitochondria and impaired autophagy. We hypothesized that changes in mitochondrial homeostasis in the AECIIs from lungs of IPF patients might compromise their survival and normal activation. We examined mitochondria in AECIIs by immunofluorescence analyses in lung sections of IPF patients $(n=7$, Supplemental Table 1; supplemental material available online with this article; doi:10.1172/JCI74942DS1) and donor age-matched controls $(n=7)$ using SP-C and ATP synthase as markers of AECIIs and mitochondria, respectively. Accumulation of mitochondrial puncta was observed in hyperplasic AECIIs from honeycomb lesions in IPF lungs compared with AECIIs from donor controls
Figure 1. Mitochondrial accumulation in AEClls from dense fibrotic areas in IPF lungs. (A) Representative immunofluorescence using anti-SP-C (AECII marker; green) and anti-ATP synthase (mitochondrial marker; red) antibodies, showing mitochondrial accumulation in hyperplasic AECIls from honeycombs in IPF lung ( $n=7$ per group). Representative cells (asterisks) are shown in detail in the insets. Scale bars: $10 \mu \mathrm{m}$. (B-E) Representative immunohistochemistry images $(n=6)$ in consecutive sections from IPF (B-D) and donor control (E) lungs using anti-SP-C and anti-TOM20 (mitochondrial marker) antibodies. Epithelial cells from honeycomb areas (B and $\mathbf{C}$ ) showed positive staining for both markers. Epithelial cells from areas with mild fibrosis (D) and donor control lung (E) showed less positive signal for the mitochondrial marker. Note the high positivity for TOM2O in macrophages located in alveolar spaces in donor control lung. Scale bars: $50 \mu \mathrm{m}$. (F) Semiquantitative scoring of SP-C/ATP synthase double-positive cells as a percentage of total SP-C-stained cells from 5 cases. MMF, mild moderated fibrosis; $\mathrm{HC}$, honeycomb; DF, dense fibrosis. Data represent mean $\pm \mathrm{SEM}$. ${ }^{*} P<0.01$ vs. normal, ${ }^{\#} P<0.01$ as indicated, 1-way ANOVA with post-hoc Bonferroni. (G) Representative images $(n=3)$ of immunohistochemistry analyses in consecutive sections from IPF lungs using BiP (ER stress marker), anti-SP-C, and anti-TOM2O antibodies. Arrows denote SP-Cpositive cells on mild fibrosis cases. Scale bars: $50 \mu \mathrm{m}$.
(Figure 1A and Supplemental Figure 1A). Immunohistochemistry analyses $(n=6)$ in consecutive lung sections of IPF lungs (using SP-C and TOM2O as markers of lung epithelial cells and mitochondria, respectively) confirmed the high mitochondrial content in AECIIs lining the areas of honeycombs and dense fibrosis (Figure 1, B and C). Conversely, AECIIs from upper lobes of IPF lungs with mild to moderated fibrosis showed lower positivity for mitochondria markers, similar to donor control lungs (Figure 1, D and E, and Supplemental Figure 1B). Semiquantitative evaluation of immunofluorescence assay for colocalization of epithelial and mitochondria markers established that AECIIs with higher mitochondrial content were located in honeycomb areas of IPF lungs ( $n=5$ per group; Figure $1 \mathrm{~F}$ ). No differences in mitochondrial content were observed in airway epithelial cells from IPF and donor control lungs (Supplemental Figure 1, C and D). These data suggested a predominant increase in mitochondrial content in AECIIs from highly fibrotic areas in IPF lungs. Mitochondrial dysfunction 
has been associated with ER stress in several neurodegenerative diseases and metabolic disorders (28-30). In addition, markers of ER stress have been found in AECIIs of IPF lungs $(8,31)$. We therefore analyzed, in consecutive lung sections, colocalization of the chaperone BiP (a marker of ER stress) and the mitochondrial marker TOM20. As reported previously, AECIIs lining honeycombs in areas of severe fibrosis were highly positive for BiP and additionally exhibited high mitochondrial content (Figure $1 G$ ). In contrast, areas of mild fibrosis in IPF lungs showed AECIIs with lower positivity for TOM2O and BiP, suggestive of an association between mitochondrial accumulation and ER stress in IPF lung AECIIs.

Ultrastructural studies confirmed the presence of abundant AECIIs from IPF patients, in marked contrast to control donor lungs ( $n=4$ per group; Figure $2 \mathrm{~A}$ ) and patients with chronic obstructive pulmonary disease (Supplemental Figure 1, E and F). Additionally, morphometric analyses of electron microscopy images demonstrated that mitochondria in IPF lung AECIIs were enlarged and dysmorphic, with significantly increased mitochondrial area and frequency of large mitochondria (Figure 2, B and C). Because AECIIs form the cell compartment that contains the majority of the lung mitochondrial mass, as described above, we therefore isolated mitochondria from IPF and control whole lungs ( $n=4-5$ per group) and assessed mitochondrial ETC complex I and IV activity. Impaired activity of both complexes was observed in mitochondria from IPF samples (mean reduction, complex I, $58 \%$; complex IV, 42\%; Figure 2D). To investigate whether the differences in mitochondrial mass and morphology in the IPF lungs were related to age, we performed ultrastructural and morphometric analyses of mitochondria in AECIIs from lungs of young $(<50$ years old) and older ( $>50$ years old) donor controls $(n=3$ per group; Figure 2E). Mitochondria in AECIIs from older lungs showed increased frequency of enlarged mitochondria as well as significantly increased mitochondrial area, but a nonsignificant trend down in number of mitochondria per cell (Figure 2, F and G). Similarly, only lungs from IPF patients showed a significant increase in the percentage of abnormal mitochondria (Figure $2 \mathrm{H}$ ), defined as swollen mitochondria with evidence of severely disrupted cristae. Mitochondrial content was also estimated by the ratio of mitochondrial to genomic DNA (mtDNA/gDNA). These analyses confirmed no significant differences in mitochondrial content between young and older control donor lungs, but a significant increase in IPF patient lungs (Figure 2I). Mitochondrial content was also analyzed in AECIIs and fibroblast cells isolated from IPF lungs versus age-matched donor controls. The mtDNA/ gDNA ratio was significantly increased in AECIIs, but not fibroblasts, of IPF lungs (Figure 2J). Thus, the increase in mitochondrial content in the IPF lung was specific to epithelial cells.

Because dysfunctional mitochondria are known to be selectively degraded by macroautophagy (mitophagy), we therefore analyzed expression of the autophagy marker LC3 in AECIIs from donor and IPF lungs by immunofluorescence assay. LC3 staining was higher in hyperplasic AECIIs from fibrotic areas in IPF lungs (Figure 3A and Supplemental Figure 2A). Analyses of confocal images using $x-z$ coordinates showed partial colocalization of LC3 and the mitochondrial marker ATP synthase (Figure 3B, arrow). Quantitative analyses of colocalization (comparing total positive pixel numbers between specific channels) showed that $30 \%$ of the pixels in the red channel (autophagy marker) appeared at the same coordinates as in the green channel (mitochondria marker). LC3 staining was negative in airway epithelial cells (Supplemental Figure 2B). Higher levels of LC3II in isolated AECIIs from IPF lungs were also observed by Western blot analyses (Figure 3C). As a second marker of autophagy, we analyzed protein levels of the adaptor protein p62 (also known as SQSTM1) in whole lysates from isolated AECIIs. p62 is degraded in cells with normal autophagy flux. Western blot analyses and immunofluorescence revealed higher p62 protein levels in AECII samples from IPF lungs compared with donor controls (Figure 3, C and D, and Supplemental Figure 2C). Finally, because lysosomes are necessary for autophagosome degradation, we conducted immunofluorescence analyses using an anti-LAMP1 antibody as a lysosome marker. AECIIs located in honeycombs from IPF lungs were variable in their lysosome content: those with high mitochondrial accumulation had low levels of LAMP1, and vice versa (Supplemental Figure 2D), similar to interstitial cells from donor control lungs. Together, these data suggest that despite the autophagy process being induced in epithelial cells from IPF lungs, autophagy flux is impaired, resulting in accumulation of dysfunctional mitochondria.

Aging and ER stress compromise mitochondrial function and fusion/fission dynamics in AECIIs of WT mice. To study the effect of aging in mitochondrial homeostasis of AECIIs, we analyzed intact primary AECIIs isolated from young (3 months) and old (18-24 months) adult C57BL/6 mice. First, to assess the effect of aging in AECII mitochondrial respiration, we measured oxygen consumption rate (OCR) and found a $40 \%$ decrease of both basal and maximal uncoupled mitochondrial respiration (the latter assessed by treatment with FCCP) in old versus young AECIIs, resulting in a significant reduction in the oxidative phosphorylation reserve capacity (Figure $4 \mathrm{~A}$ ). This was consistent with the potential inability of old mitochondria to respond to elevated energy demands. Addition of the ATP synthase inhibitor oligomycin allowed us to measure oxygen consumption coupled to ATP production. Between young and old AECIIs, the link of respiration to ATP production was comparable (Figure 4A). Oxygen consumption was inhibited in young and old AECIIs by the mammalian complex I antagonist rotenone (data not shown), which confirmed that this measurement specifically reported mitochondrial respiration. To confirm that our observations were not strain dependent, we performed studies of mitochondrial respiration in young and old AECIIs from BALB/c mice. Similar to our studies in $\mathrm{C} 57 \mathrm{BL} / 6$ mice, old AECIIs from BALB/c showed decreased basal and maximal mitochondrial respiration (Supplemental Figure 3A). ER stress is a common finding in AECIIs from IPF lungs and in aging mice with lung fibrosis $(8,9,11)$. To evaluate whether ER stress causes mitochondrial dysfunction, we measured mitochondrial respiration in primary isolated AECIIs from young and old mice exposed to tunicamycin (TM; $1 \mu \mathrm{g} / \mathrm{ml}$ for 1 hour), a well-known ER stress stimulator. TM treatment significantly diminished basal OCR in both young and old AECIIs compared with untreated cells (young, 6-fold; old, 13-fold; Figure 4A), indicative of higher susceptibility to ER stress-induced mitochondrial damage. Furthermore, maximal respiration in aging mitochondria was reduced more than $50 \%$ (Figure $4 \mathrm{~A}$ ), indicative of a low capacity to respond to higher energy demands. 

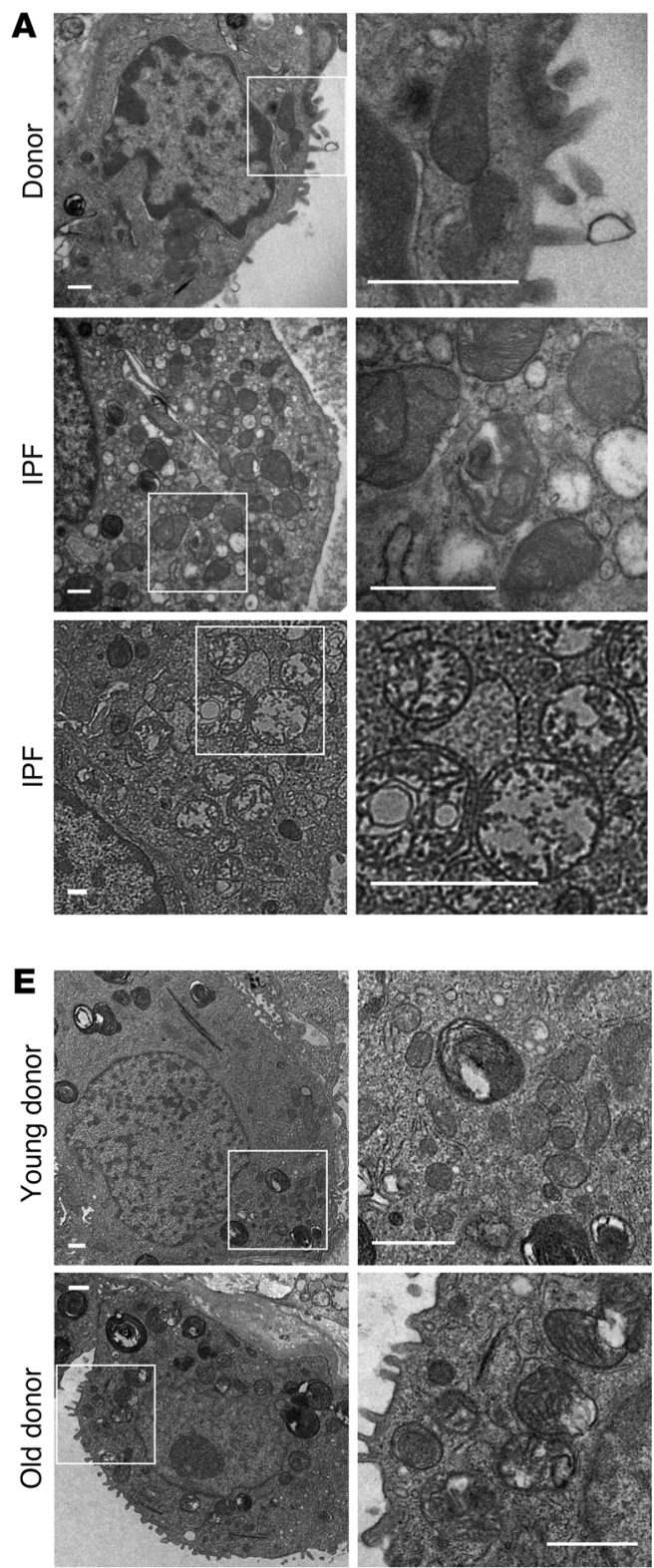

G

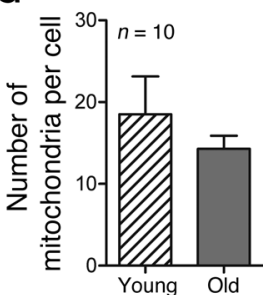

H

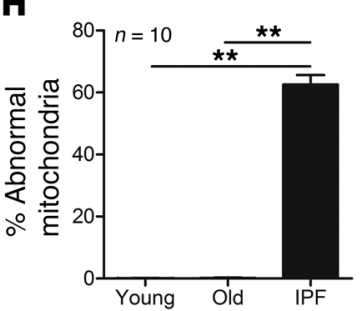

B
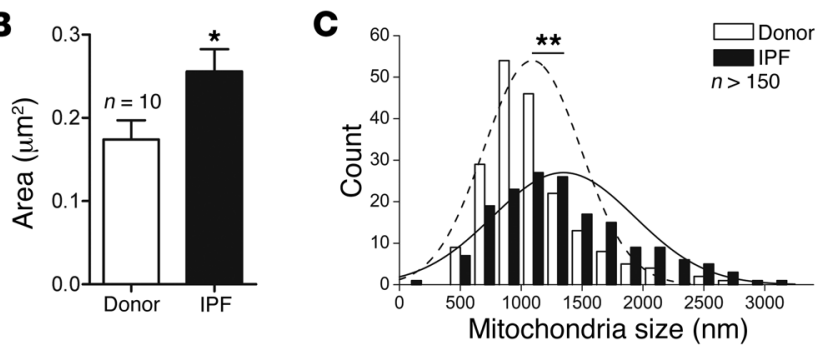

D
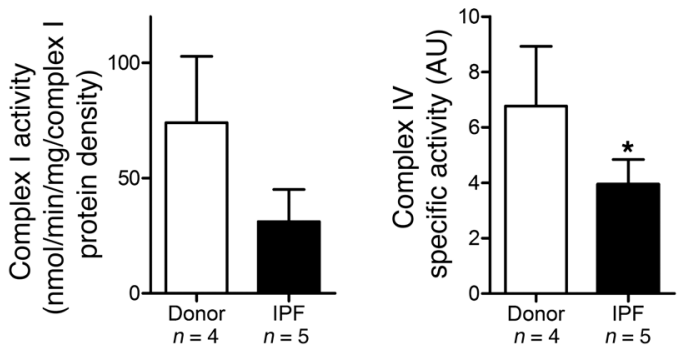

$\mathbf{F}$
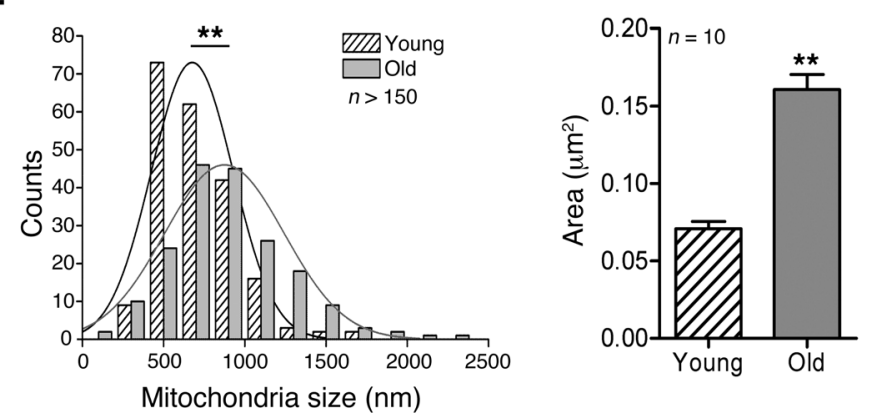
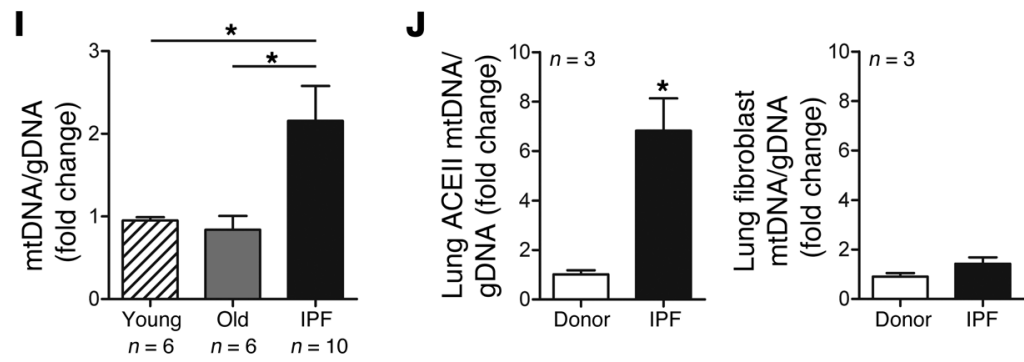

Figure 2. Accumulation of dysmorphic and dysfunctional mitochondria in AECIls from IPF lungs. (A) Representative TEM ( $n=4$ per group) in donor and IPF AECIIs (identified by the presence of lamellar bodies). Boxed regions are shown enlarged at right. Scale bars: $500 \mathrm{~nm}$. (B) Quantitative analyses of morphometric data from TEM images. (C) Frequency of mitochondria sizes in donor control and IPF lungs obtained from TEM images. (D) Isolated lung mitochondria showed reduced mitochondrial complex I and complex IV activity in IPF patients relative to age-matched donor controls. (E) Representative TEM ( $n=3$ per group) in AECIls from young ( $<50$ years) and older ( $>50$ years) donor control lungs. Boxed regions are shown enlarged at right. Scale bars: $500 \mathrm{~nm}$. (F) Frequency of mitochondria sizes and mitochondrial area from AECIls in young and old donor controls (obtained from TEM images). (G) No significant difference in number of mitochondria per cell (obtained from TEM images) between young and old donor control AEClls. (H) Percentage of abnormal mitochondria (swollen with evidence of severely disrupted cristae over all mitochondria) (obtained from TEM images). (I) Increased mitochondrial mass in IPF lungs, assessed by mtDNA/gDNA ratio. (J) Mitochondrial mass, assessed by mtDNA/gDNA ratio, in isolated AECIls and lung fibroblasts from donor and IPF lungs. Data represent mean $\pm \operatorname{SEM}(\mathbf{B}, \mathbf{D}$, and $\mathbf{F}-\mathbf{J}) .{ }^{*} P<0.05,{ }^{* *} P<0.01$, unpaired, 2-tailed Student's $t$ test (B-D, F, G,and J) or 1-way ANOVA with post-hoc Bonferroni ( $\mathbf{H}$ and $\mathbf{I})$. 
A
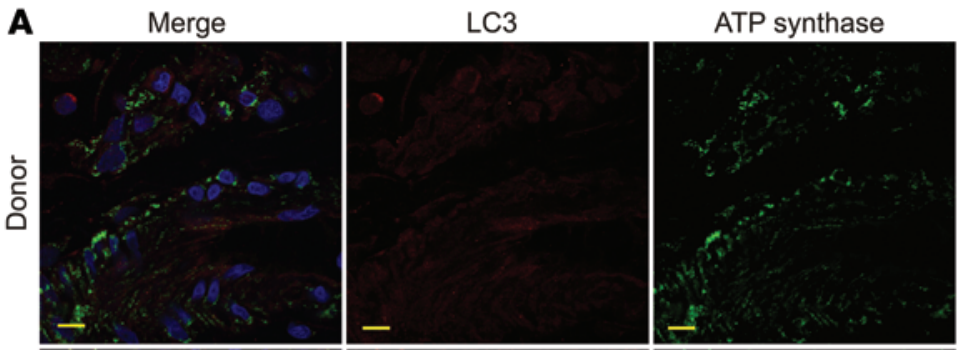

늠
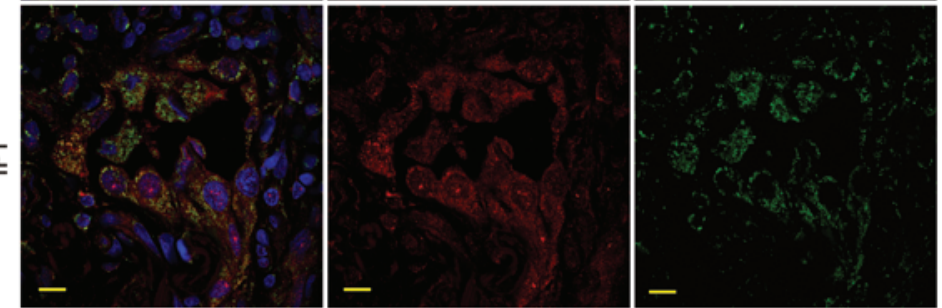

B

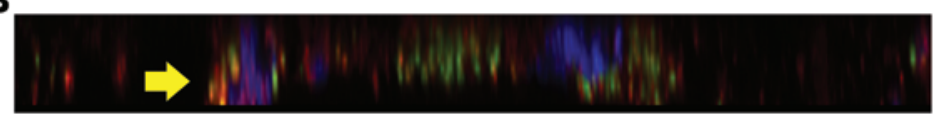

C
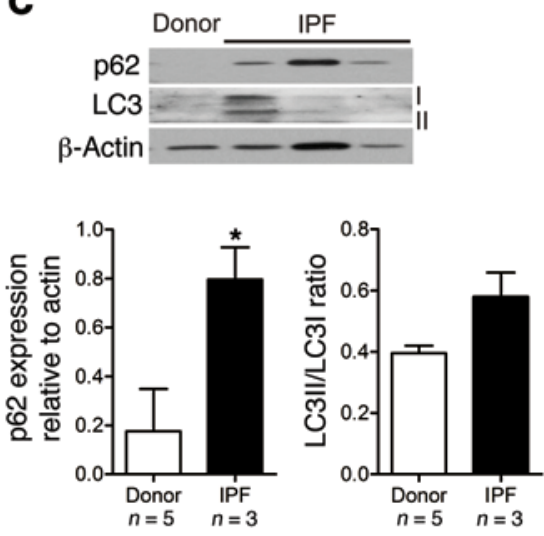

D

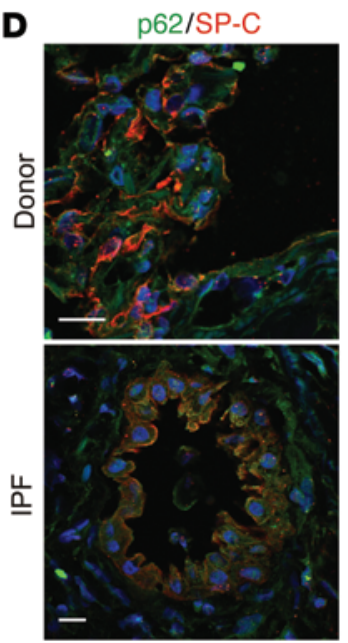

Figure 3. Defective autophagy in AECIls from IPF lungs. (A) Representative immunostaining of lung sections from donor and IPF patients using anti-LC3 (red; autophagosomal marker) and anti-ATP synthase (green; mitochondrial marker) antibodies. Yellow puncta denote colocalization. Scale bars: $10 \mu \mathrm{m}$. (B) $x-z$ coordinate image of $z$ stack of merged LC3 and ATP synthase image of the IPF lung section in A. Partial colocalization was seen for the mitochondrial and autophagosomal markers (arrow). (C) Western blot analyses of p62 and LC3I/LC3II in isolated AECIls from donor age-matched control and IPF lungs. Each lane represents an individual AECII preparation. Blots were stripped and reblotted using an anti- $\beta$-actin antibody as loading control. Results are also quantified below. Data represent mean \pm SEM. ${ }^{*} P<0.05$, unpaired, 2-tailed Student's $t$ test. (D) Representative immunostaining of donor and IPF patient lung sections using anti-SP-C (green) and anti-p62 (red). Yellow indicates colocalization of the markers. Scale bars: $10 \mu \mathrm{m}$.

Increased mitochondrial size can be associated with alterations in mitochondrial dynamics (32); moreover, mitochondrial fusion might inhibit the mitophagy process, causing mitochondrial accumulation in the cells (33). Expression analyses of mitochondrial fusion/fission dynamic modulators showed that TM treatment in young mice inhibited mitochondrial fission via phosphorylation of Ser637 of DRP1 and upregulation of the fusion modulators OPA1 and mitofusin 1 (MFN1) (Figure 4, G and $\mathrm{H})$. A predominant mitochondrial fusion dynamic was also observed in lungs of naive old mice, as shown by phosphorylation of DRP1 as well as enhanced expression of OPA1 and MFN1. The same expression pattern was observed in old lungs in the presence of TM treatment, with MFN2 additionally increased (Figure 4, G and H). Collectively, these findings suggested that mitochondrial function and dynamics are altered in aging AECIIs.

Alteration in mitophagy flux in aging and stressed AECIIs. To confirm that ER stress impaired mitochondrial homeostasis in lung epithelial cells, we treated A549 cells with TM and measured mitochondrial mass

To investigate whether mitochondrial dysfunction in mouse aging AECIIs is associated with changes in mitochondrial morphology, we performed ultrastructural analyses. Electron micrographs revealed that mitochondria in AECIIs from old animals had preserved cristae structure and normal matrix density, but the frequency of larger mitochondria was higher in AECIIs from old animals (Figure 4, B and C). We next evaluated whether ER stress perturbs mitochondrial morphology, using electron microscopy to analyze morphological changes in mitochondria in lungs of mice exposed in vivo to TM $(2 \mu \mathrm{g} /$ mouse). Stimulation of ER stress was confirmed by splicing of XBP1 and phosphorylation of JNK at day 15 after treatment (Supplemental Figure 3, B-E). TM induced comparable mitochondrial enlargement in AECIIs from young and old mice, as shown by higher frequencies of large mitochondria and increased mitochondrial area (Figure 4, D-F). In AECIIs from old lungs, TM treatment increased mitochondrial area and the percentage of abnormal mitochondria (Figure 4, $\mathrm{D}-\mathrm{F}$ ), defined as swollen mitochondria with evidence of reduced matrix density and cristae. and mitochondrial membrane potential by MitoTracker dye and the potentiometer dye JC-1, respectively. ER stress stimulation enhanced mitochondrial mass, and this was diminished by starvation-induced autophagy. Conversely, the autophagy inhibitor bafilomycin A1 significantly increased mitochondrial accumulation (Figure 5A). TM also reduced mitochondrial membrane potential (which was further diminished with bafilomycin A1 treatment), while increasing the percentage of apoptotic alveolar epithelial cells (Figure 5, B and C).

Mitophagy and mitochondrial biogenesis have a substantial effect on mitochondrial function and homeostasis in aging AECIIs. Expression levels of the mitochondrial biogenesis marker PPAR $\gamma$ coactivator $1 \alpha$ (Ppargc1a), the mitochondrial transcription factor A (Tfam), and the mitochondrial gene cytochrome c (Cycs) were comparable between young and old murine lungs (Supplemental Figure 4, A and B), suggestive of similar mitochondrial biogenesis as well as mtDNA replication and transcription. We next analyzed mitochondrial mass by assessing levels of the mitochondrial protein TOM20 and the autophagy markers p62 and LC3II in 


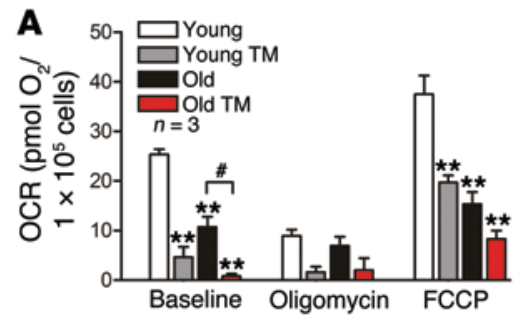

B
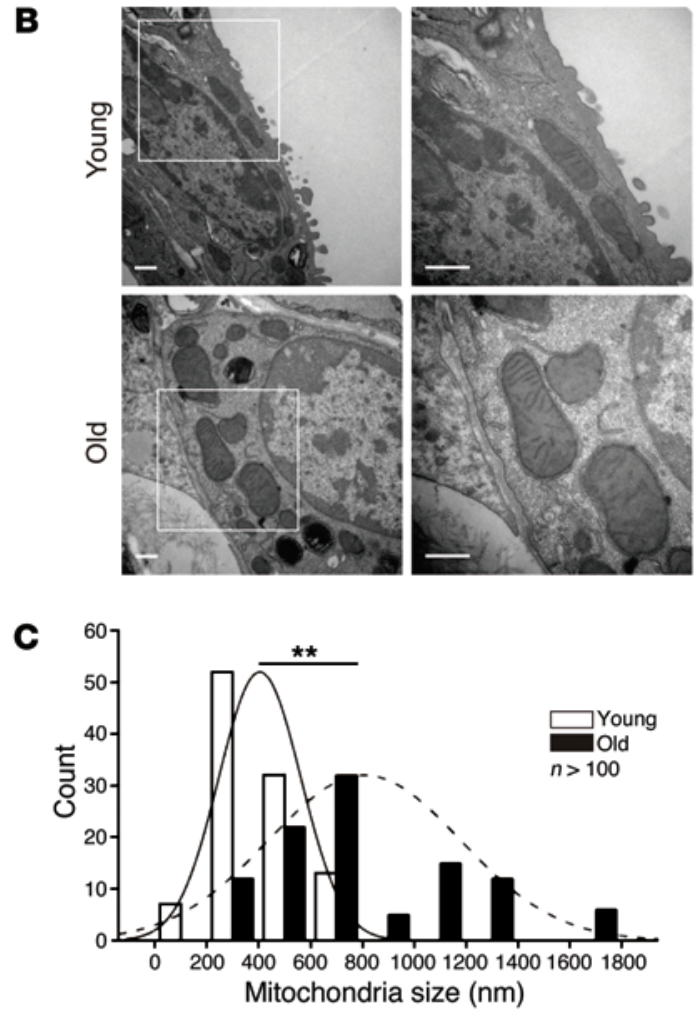

D

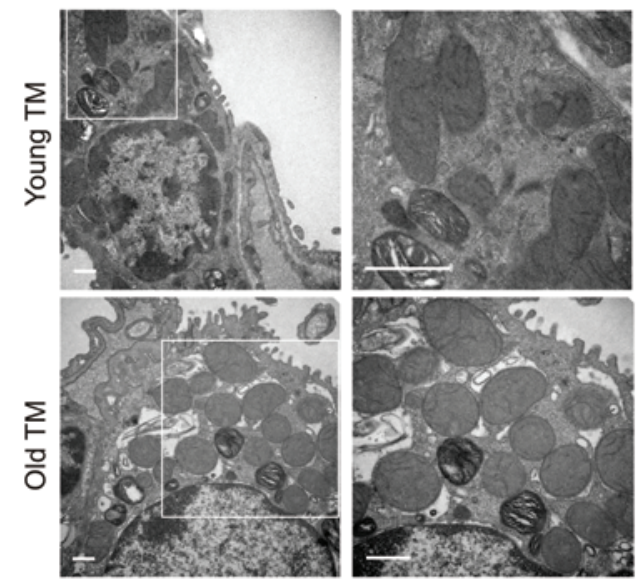

E

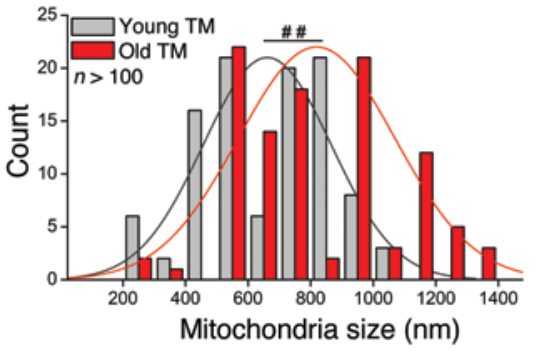

$\mathbf{F}$
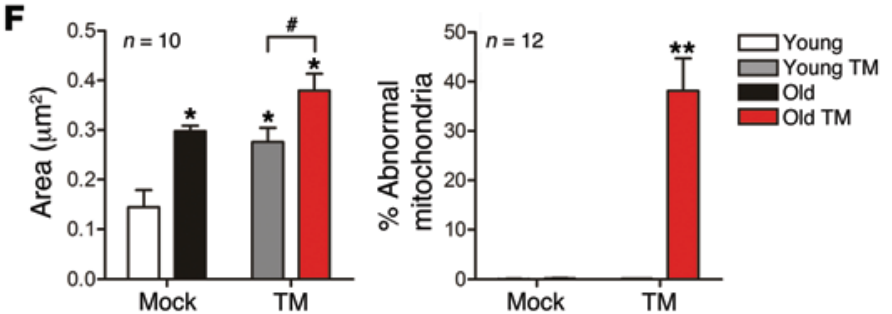

G

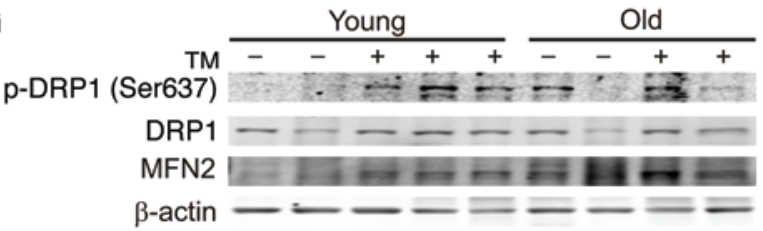

H
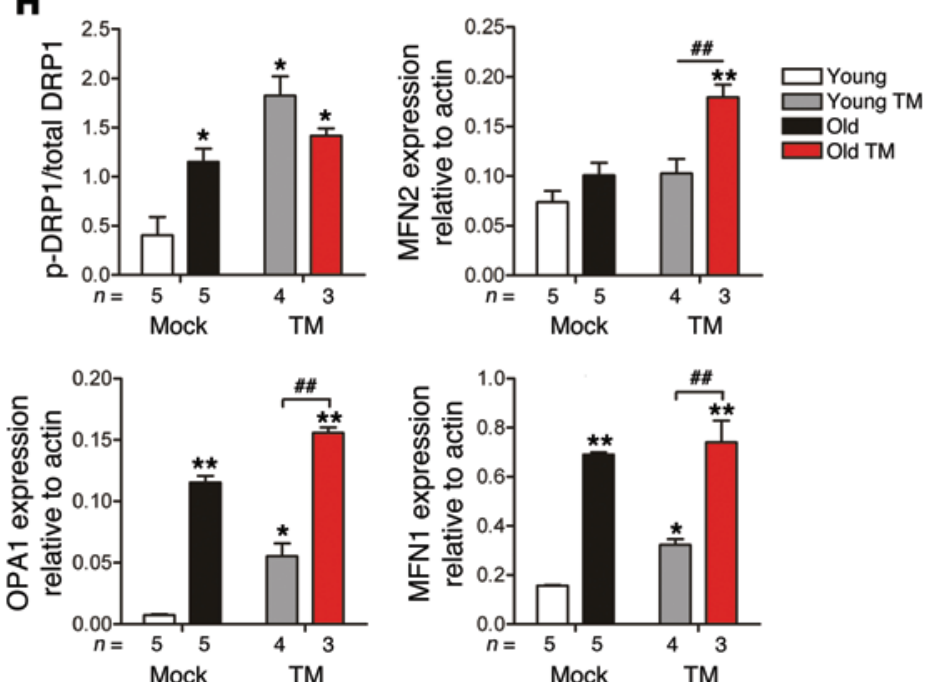

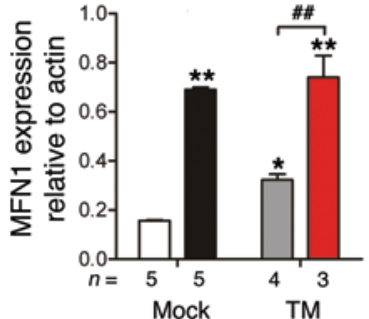

Figure 4. Impaired mitochondrial function and fission/fusion dynamics in AEClls with age. (A) Mitochondrial respiration parameters in isolated primary AECIls from young and old C57BL/ 6 mice with vehicle control or TM treatment $(1 \mu \mathrm{g} / \mathrm{ml}$ for 1 hour). (B) Representative TEM ( $n=3$ per group) showing enlarged mitochondria but preserved structure in AECIls from naive old mice. Boxed regions are shown enlarged at right. Scale bars: $500 \mathrm{~nm}$. (C) Quantitative morphometry showed significantly increased frequency of large mitochondria in AEClls from naive old mice ( $n \geq 100$ per condition). (D) Representative TEM ( $n=3$ per group) showing enlarged mitochondria in AECIls from TM-treated young and old mice. Boxed regions are shown enlarged at right. Scale bars: $500 \mathrm{~nm}$. (E) Morphometry showed increased frequency of large mitochondria in young and old AECIls after TM treatment ( $n \geq 100$ per condition). (F) Area of AECII mitochondria and percentage of abnormal mitochondria (swollen with evidence of severely disrupted cristae over all mitochondria) from TEM images. (C) Representative Western blot membranes showing higher expression levels of mitochondrial fusion modulators ( $p$-DRP1, MTF2, OPA1, and MTF1) in lungs from young and old mice treated with TM. (H) Density analyses of Western blots for fusion and fission mitochondrial modulators. Data represent mean $\pm S E M(\mathbf{A}, \mathbf{F}$, and $\mathbf{H}) .{ }^{*} P<0.05$, ${ }^{* *} P<0.01$ vs. young; ${ }^{\#} P<0.05,{ }^{\# \#} P<0.01$ as indicated, 2 -tailed Student's $t$ test $(\mathbf{A}, \mathbf{C}$, and $\mathbf{E})$ or 1-way ANOVA with post-hoc Bonferroni $(\mathbf{F}$ and $\mathbf{H})$. 

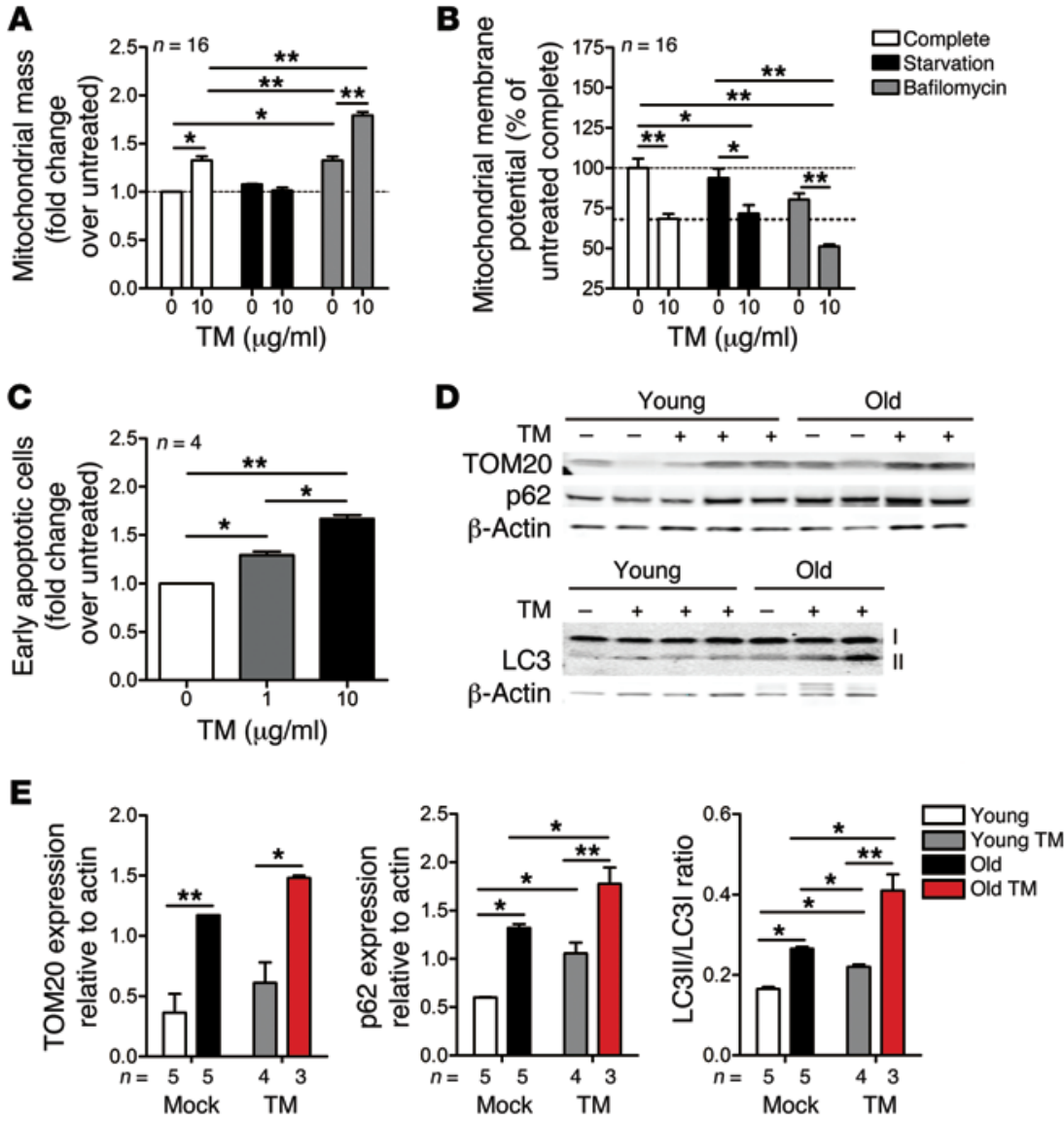

Figure 5. Stimulation of ER stress deteriorates mitochondrial function and impairs mitophagy in lung epithelial cells. (A) A549 cells were treated with or without TM ( $1 \mu \mathrm{g} / \mathrm{ml}$ for 24 hours), and mitochondrial mass was determined by MitoTracker Green. Induction of autophagy by serum starvation reduced mitochondrial mass in TM-treated cells. The autophagy inhibitor bafilomycin $\mathrm{A} 1$ increased mitochondrial mass in untreated and TM-treated cells. (B) TM induced dose-dependent depolarization of mitochondria in A549 cells (assessed by JC-1 dye staining). Depolarization was increased in the presence of bafilomycin A1, but was not affected by starvation conditions. (C) Increased doses of TM induce apoptosis of A549 cells (assessed by annexin V staining). (D) Representative Western blot analyses showing increased levels of the mitochondrial marker TOM20 and autophagy markers p62 and LC3I/LC3II in lung lysates from aging and young mice after vehicle and TM treatment ( $2 \mu \mathrm{g} /$ mouse). The $\beta$-actin blot was obtained from parallel samples run on a separate gel from the TOM20 and p62 blots. (E) Density analyses of Western blots in $\mathbf{D}$. Data represent mean \pm SEM (A-C and E). ${ }^{*} P<0.05,{ }^{* *} P<0.01,1-(\mathbf{A}-\mathbf{C})$ or 2-way (E) ANOVA with post-hoc Bonferroni. lungs from young and old mice. We found a significant increase of mitochondrial mass after TM treatment as well as in lung samples from old mice (Figure 5, D and E). Additionally, age and ER stress resulted in elevation of LC3II and p62 (Figure 5, D and E), similar to our findings in IPF lungs and consistent with blockade of the autophagy flux. Because ER stress is a known inducer of autophagy, we analyzed autophagy induction in vivo by intratracheal instillation of TM (20 $\mu \mathrm{g} /$ mouse) together with chloroquine (2 $\mathrm{mg} / \mathrm{kg}$ weight), which inhibits autophagy by preventing lysosomal acidification. We found that TM caused significant accumulation of LC3 in lung cells from old mice 4 hours after injection, but not in young lung cells (Supplemental Figure 4C), which suggested that old mice also have increased induction of autophagy in response to acute ER stress stimulation.

$E R$ stress recapitulates age-related impairment of mitochondria and susceptibility to lung fibrosis in young animals. We previously showed that infection with MHV68 (a murine gammaherpesvirus homologous to EBV) induces ER stress in AECIIs and lung fibrosis in aging mice, whereas young mice are protected (11). To examine whether ER stress in young mice recapitulates susceptibility to lung fibrosis, we pretreated young mice with a single intratracheal dose of TM ( $2 \mu \mathrm{g} /$ mouse) 48 hours before MHV68 infection. Ultrastructural analyses of mitochondria in AECIIs showed larger mitochondrial profiles and number of mitochondria per cell in AECIIs from old and TM-pretreated young infected mice (Figure 6, A-D). Quantification of mtDNA (15 days after MHV68 infection) showed significantly increased mitochondrial mass in old and young
TM-pretreated infected mice (Figure 6E). Similar to our previous studies, lung pathology (analyzed by Masson trichrome staining) showed mild pneumonitis in young infected mice and severe interstitial fibrosis in old infected animals (Figure 6F). TM pretreatment of young mice led to a greater extent of lung fibrosis development upon MHV68 infection, compared with young infected mice without TM (Figure 6F). The worsening of lung pathology by TM pretreatment correlated with a significant increase of collagen deposition upon infection, as assessed by increased levels of hydroxyproline and the profibrotic factor $\mathrm{Tg} f b$ (Figure 6, G and $\mathrm{H}$ ).

PINK1 expression in IPF lungs is low and decreases with age and ER stress. Our present data showed that ER stress and aging mediated changes in mitochondrial dynamics, function, and turnover. PINK1 is a key regulator of mitochondrial quality control, including mitochondrial respiration, mitochondrial dynamics, and mitophagy (19). Microarray analyses showed significant downregulation of PINK1 in lung samples from IPF patients (Figure 7A and Supplemental Table 2). Low expression of PINK1 in IPF lungs was confirmed by real-time RT-PCR in total and isolated AECII samples (Figure 7, B and C). Western blot analyses of PINK1 in AECII lysates showed absence of full-length PINK1 in IPF samples (Figure 7D and Supplemental Figure 5A). In contrast, transcript and protein levels of PINK1 in IPF primary lung fibroblasts were similar to those of young ( $<50$ years) and old ( $>50$ years) donor controls (Figure 7, E and F, and Supplemental Figure 5B). Expression of PINK1 was also analyzed in mouse lung samples. Pink1 mRNA levels were significantly diminished in aging versus naive young 

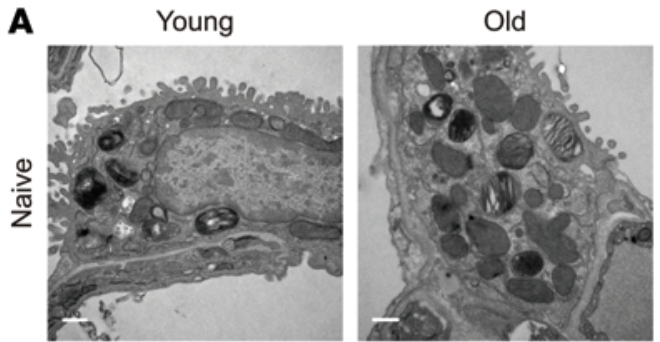

E
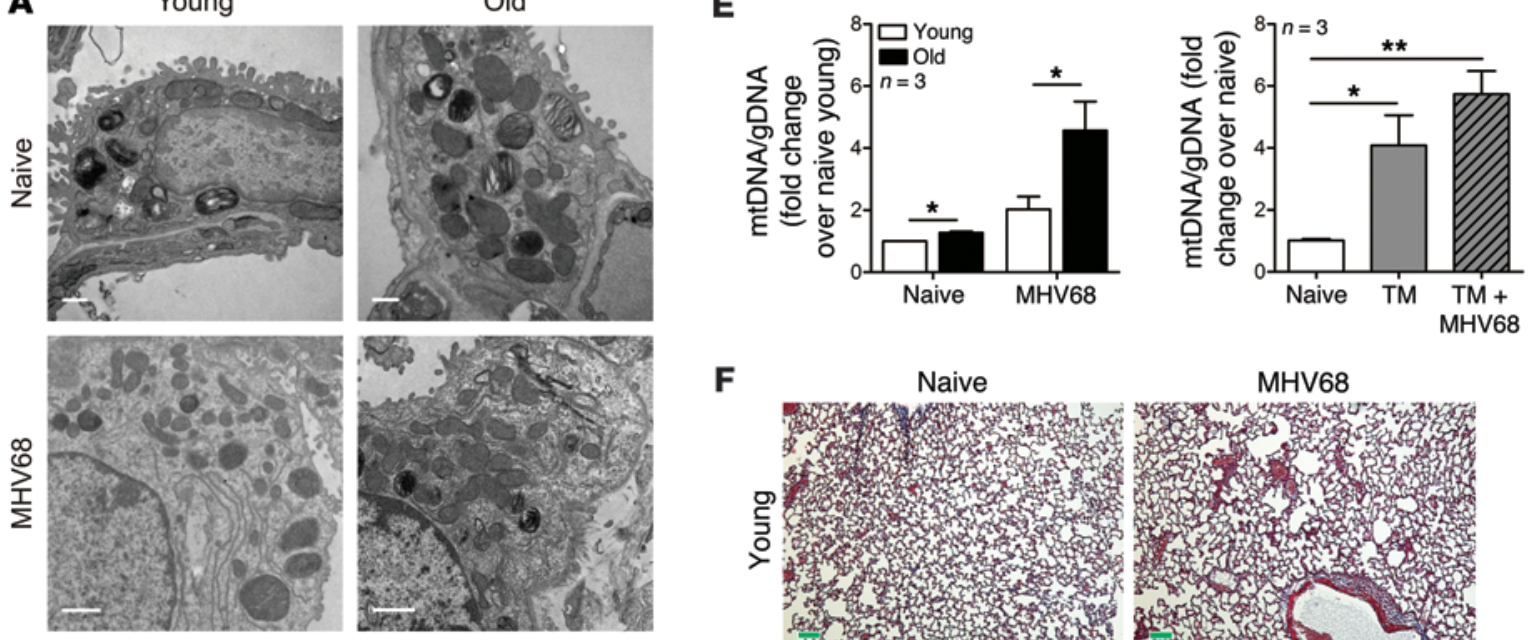

\section{$\mathbf{F}$}

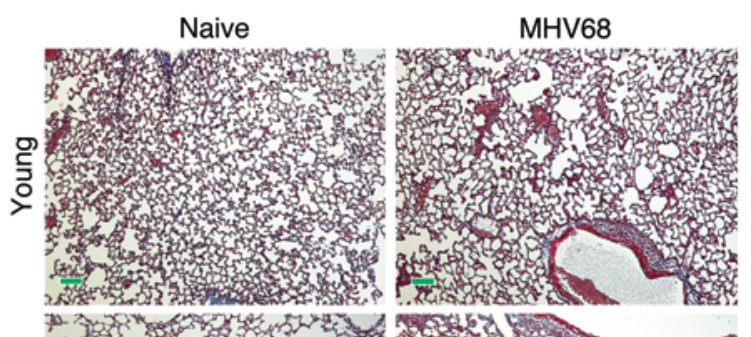

B
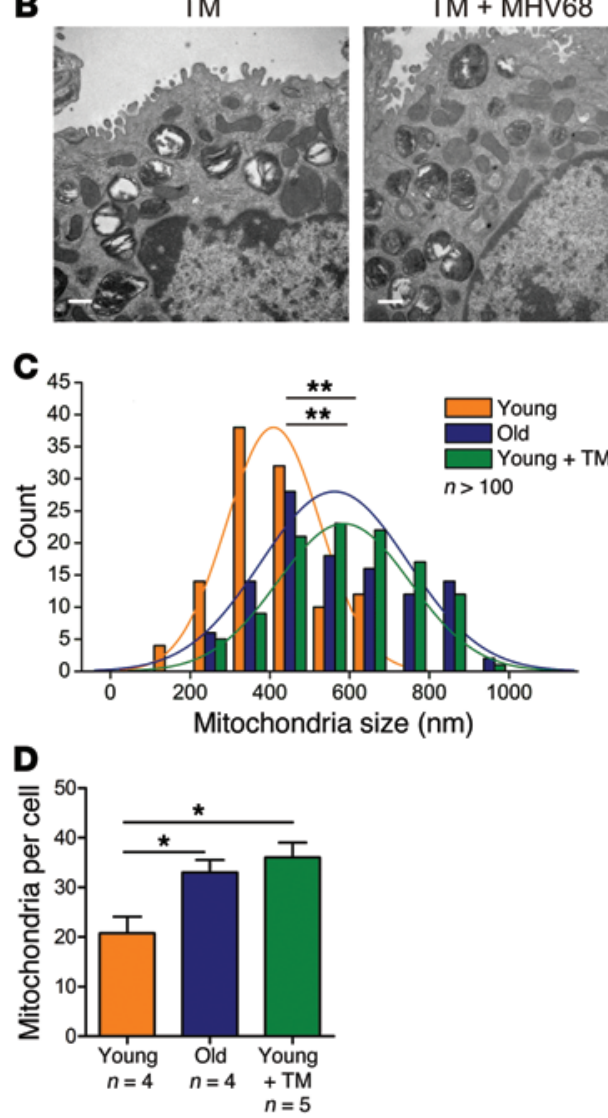

$\mathrm{TM}+\mathrm{MHV} 68$
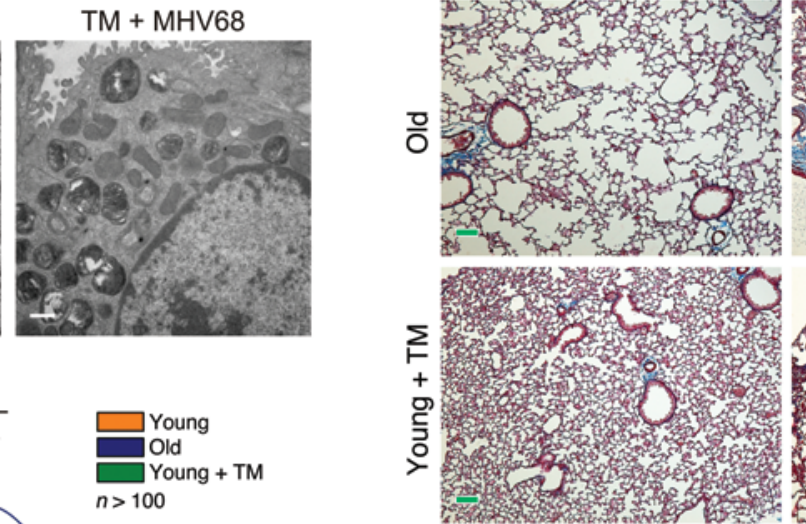

G

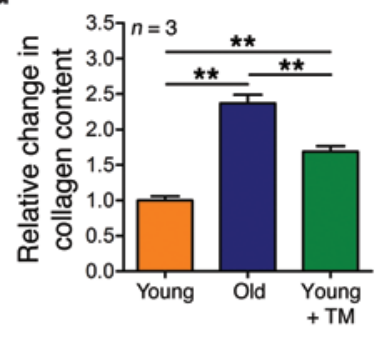

H

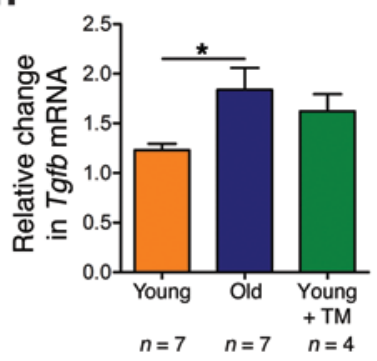

Figure 6. ER stress stimulation recapitulates aging-associated susceptibility to lung fibrosis. (A) Representative TEM $(n=4)$ of lungs from young and old mice at 0 (naive) and 15 days after MHV68 infection. Scale bars: $500 \mathrm{~nm}$. (B) Representative TEM $(n=4)$ of AECIls from young mice treated with TM only $(2 \mu \mathrm{g} /$ mouse) or TM ( $2 \mu \mathrm{g} /$ mouse) followed by MHV68 infection 48 hours later. Scale bars: $500 \mathrm{~nm}$. (C) Quantitative morphometry showed increased frequency of large mitochondria in young infected AECIls when pretreated with TM. (D) Number of mitochondria per AECII (from TEM images) of MHV68infected mice. Old mice and young TM-pretreated mice showed high numbers of mitochondria. (E) mtDNA/gDNA in lung samples showed increased mitochondrial mass after MHV68 infection in old mice and with TM treatment in young mice. (F) Representative Masson trichrome staining from lungs at day 15 after MHV68 infection, showing increased pneumonitis and collagen deposition (blue) in old mice and young TM-pretreated mice. Scale bars: $50 \mu \mathrm{m}$. (G) Relative change in collagen deposition (assessed by hydroxyproline level) upon MHV68 infection. (H) Relative change in Tgfb transcription upon MHV68 infection. Data represent mean \pm SEM (D, E, G, and $\mathbf{H}) .{ }^{*} P<0.05,{ }^{* *} P<0.01$, 2-tailed Student's $t$ test (C) or 1- (D, E, right, G, and $\left.\mathbf{H}\right)$ or 2-way (E, left) ANOVA with post-hoc Bonferroni. 


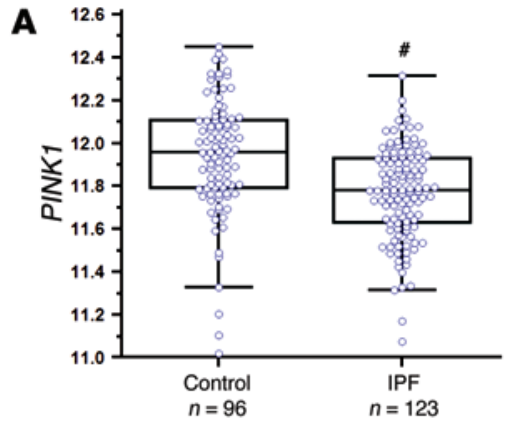

B

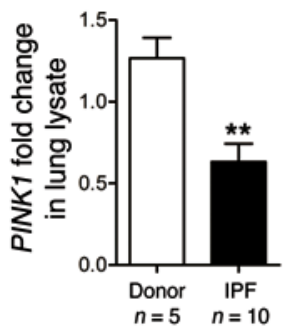

C

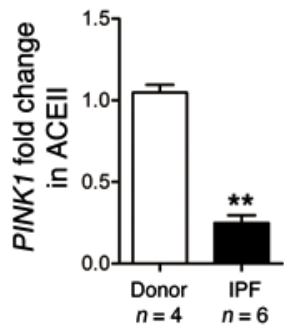

D

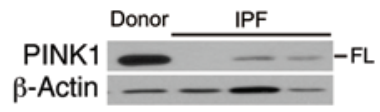

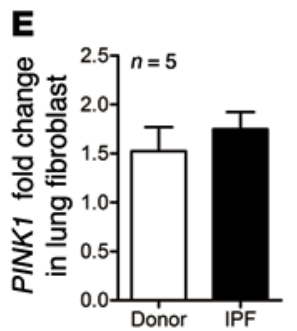

$\mathbf{F}$
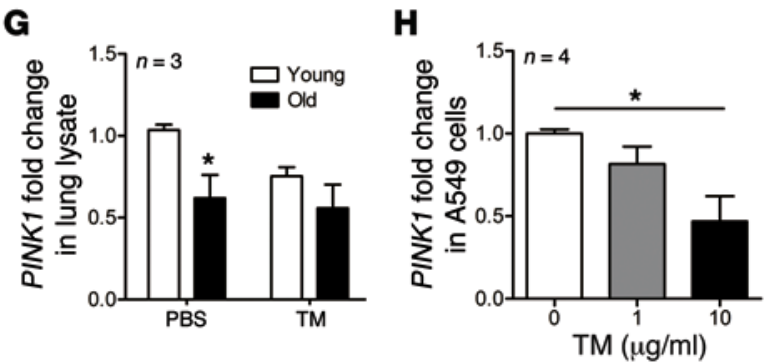

Figure 7. Downregulation of PINK1 expression in AECIIs and lungs from aging and TM-treated mice. (A) Microarray analyses of the LGRC cohort showed significantly decreased PINK1 expression in IPF patients ( $P<0.0001 \mathrm{vs.} \mathrm{control).} \mathrm{Data} \mathrm{are} \mathrm{presented} \mathrm{as} \mathrm{box-and-whisker} \mathrm{plots,} \mathrm{with} \mathrm{horizontal} \mathrm{bars} \mathrm{rep-}$ resenting medians, top whisker representing maximal expression, and bottom whisker representing the 5th percentile. (B) Significant reduction of PINK1 transcripts, assessed by quantitative RT-PCR, in whole IPF lungs. (C) Significant reduction of PINK1 transcripts in isolated IPF AECIIs. (D) Immunoblot of lysates of isolated AEClls from donor control and IPF lungs showing lower levels of full-length (FL) PINK1 in IPF lungs. (E) PINK1 transcript levels, assessed by quantitative RT-PCR, in isolated lung fibroblasts from donor control and IPF patients. (F) Representative immunoblot of isolated lung fibroblasts from young ( $<50$ years) and old ( $>50$ years) donor controls and IPF patients, showing similar protein levels of full-length PINK1 and isoforms $\Delta N 1$ and $\Delta N 2$. (C) Quantitative RT-PCR showed diminished Pink1 expression in murine lungs with age and after TM treatment. (H) In vitro TM treatment diminished PINK1 expression in A549 cells. Data represent mean \pm SEM (B, C, E, G, and $\mathbf{H}) .{ }^{*} P<0.05,{ }^{* *} P<0.01$, unpaired 2-tailed Student's $t$ test (B, C, and E) or $1-(\mathbf{H})$ or 2-way (G) ANOVA with post-hoc Bonferroni.

lungs, as well as in lungs from young mice after in vivo treatment with TM $(2 \mu \mathrm{g} /$ mouse; Figure $7 \mathrm{G})$. In agreement with these observations, PINK1 transcripts in A549 cells were also downmodulated with increasing doses of TM (Figure 7H).

Low PINK1 expression causes mitochondrial depolarization and profibrotic factor expression in AECIIs. To examine whether changes in PINK1 expression could cause the mitochondrial accumulation and depolarization observed with ER stress, A549 cells were transfected with PINK1 shRNA (Supplemental Figure 6A) and exposed to TM and bafilomycin A1 to induce ER stress and block the autophagy flux, respectively. Although knockdown of PINK1 expression did not itself increase mitochondrial mass, a significant increase was observed in PINK1-knockdown cells after TM and bafilomycin A1 treatment (Figure 8A), which supported the notion that PINK1 contributes to mitochondrial turnover mainly during cell stress. In contrast, PINK1 knockdown alone diminished mitochondrial membrane polarization, and this was further decreased with TM and bafilomycin A1 treatment (Figure 8A). PINK1 overexpression ameliorated the TM- and bafilomycin A1-induced mitochondrial accumulation and depolarization (Figure 8B and Supplemental Figure 6B). Cell viability was significantly reduced by PINK1 knockdown, and was further decreased by TM and bafilomycin A1 treatment; conversely, PINK1 overexpression improved cell survival, even in the presence of TM or bafilomycin A1 (Supplemental Figure 6, C and D).

We next analyzed expression of proinflammatory and profibrotic mediators in PINK1-knockdown lung epithelial cells. A549 cells exhibited 1.5- and 2-fold increases of TGFB and FGF2 mRNA, respectively, with shRNA-mediated loss of PINK1 expression compared with scrambled shRNA control (Figure 8, C and D). In cells exposed to exogenous mtDNA, mRNA levels of TGFB, FGF2, and IL6 increased 1.7- to 1.9-fold compared with the respective controls (Figure 8, C and D, and Supplemental Figure 6E).

PINK1 is required for mitochondrial integrity in AECIIs and resistance to lung fibrosis. To further confirm the role for PINK1 in mitochondrial homeostasis in AECIIs, we performed ultrastructural analyses of lungs from 2- to 3-month-old mice deficient in PINK1 and WT littermate controls ( $n=3$ per group; Figure 9A). Pink1 $1^{-/}$mice exhibited frequent enlarged swollen mitochondria, as shown by TEM images and morphometric determinations of frequency of large mitochondria, mitochondrial area, and percentage of abnormal swollen mitochondria (Figure 9, A and B). The percentage of abnormal mitochondria was similar to that in IPF patient versus donor control lungs (Supplemental Figure 7A). The increased size and swollen morphology in PINK1-deficient (Pink1 $1^{+-}$and Pink1 $^{-/-}$) mitochondria were associated with lower numbers of mitochondria per cell, but no significant difference in mtDNA content, compared with $\mathrm{Pink1}^{+/+}$littermate controls (Figure 9C). Since PINK1 deficiency has been associated with low biosynthesis of mtDNA (34), we measured the percentage of AECII cytoplasm occupied by mitochondria as an indicator of mitochondrial mass and found that mitochondrial area per cell was significantly higher in $\mathrm{Pink1}^{-/-}$versus $\mathrm{Pink} \mathrm{I}^{+/+}$mice (Figure 
A

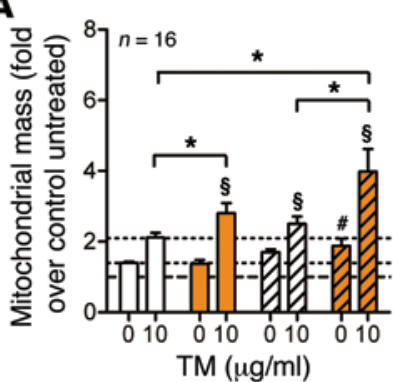

B

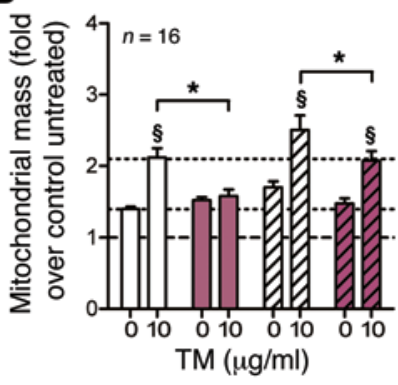

C

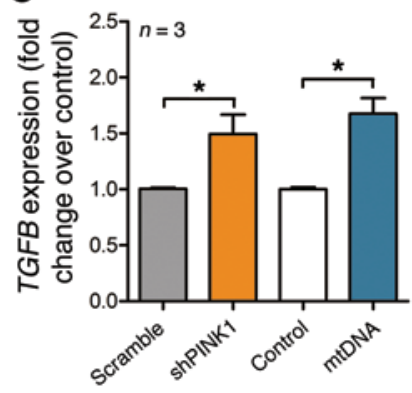

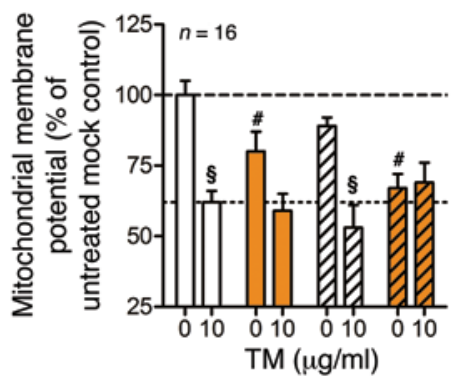

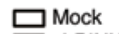

Mock+ bafilomycin ZZ shPINK1 + bafilomyci

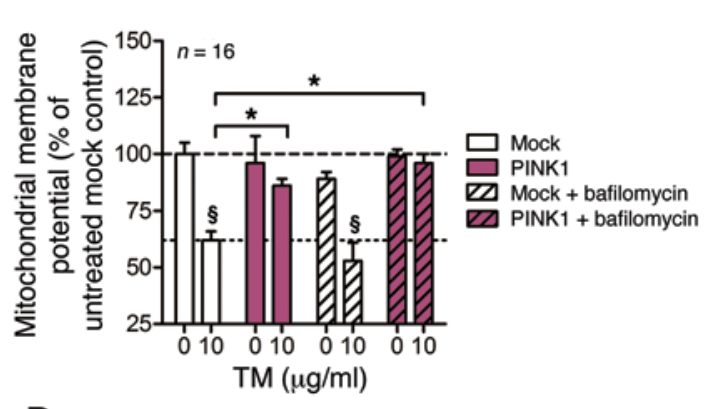

D

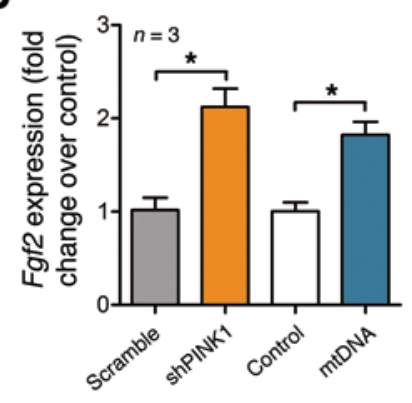

Figure 8. PINIK1 modulates mitochondrial homeostasis. (A) Mitochondrial mass and depolarization in A549 cells transfected with scramble control (Mock) or PINK1 shRNA (shPINK1), assessed by MitoTracker Green FM and JC-1 staining, respectively. Downmodulation of PINK1 was detrimental upon TM treatment 48 hours after transfection, inducing a higher accumulation of depolarized mitochondria. Bafilomycin A1 (10 nM) further increased mitochondrial numbers and depolarization. Data represent mean \pm SEM of 16 replicates per condition. (B) Mitochondrial mass and depolarization induced by TM and/ or bafilomycin A1 treatment was improved by PINK1 overexpression. Data represent mean \pm SEM of 16 replicates per condition. (C and D) Increased levels of TCFB (C) and FGF2 (D) mRNA in A549 cells after PINK1 downregulation by shRNA or after treatment with mtDNA. (A-D) Data represent mean \pm SEM. $\S P<0.05$ vs. respective no-TM control, ${ }^{\#} P<0.05$ vs. no-TM Mock; ${ }^{*} P<0.05$ as indicated, 1-way ANOVA with post-hoc Bonferroni.
9C). Mitochondrial morphology in $\mathrm{Pink1}^{+/-}$mice was normal at 2-3 months of age, but the size and number of mitochondria per cell increased significantly with age; at 9 months, the number of mitochondria per cell was 2-fold higher in Pink1 ${ }^{+/-}$versus $\mathrm{Pink1}^{+/+}$ AECIIs (Figure 9B and Supplemental Figure 7B). Pathological analyses of the lungs using trichrome staining showed increased collagen deposition around airways and alveolar septa in Pink1 ${ }^{-/-}$ compared with $\mathrm{Pink1}^{+/+}$mice (Figure 9D). Remodeling in Pink1/lungs was confirmed by measurement of collagen deposition, specifically by the increased hydroxyproline levels and transcript levels of Col1a1 (Figure 9E). Electron microscopy analyses showed numerous fibers of collagen within the alveolar walls and surrounding AECIIs, similar to areas of mild fibrosis observed in IPF lungs (Figure 9F and Supplemental Figure 7C). Quantitative RT-PCR (qRT-PCR) analyses for transcript levels of profibrotic growth factors and cytokines showed higher expression of $\mathrm{Tg} f b$, Fgf2, and Tnfa in naive PINK1-deficient versus control lungs (Supplemental Figure 7D).

We then examined mitochondrial function in lungs from PINK1-deficient and control mice ( $n=3$ per group) by assessing of mitochondrial ETC complex I and IV activity. Impaired activity of complex I (mean reduction, 42\%) was observed in mitochondria from naive $\mathrm{Pink1}^{+/-}$and $\mathrm{Pink1} 1^{-/-}$mice (Figure 10A). In parallel, we subjected Pink1 ${ }^{+/+}, \mathrm{Pink1}^{+/-}$, and Pink1 $1^{-/-}$littermate mice (3-9 months old) to infection with MHV68 (a strong inducer of
ER stress and potential second hit leading to the development of lung fibrosis in the setting of a vulnerable AECII), as previously described (11, 31, 35-39). First, we demonstrated evident mitochondrial dysfunction in Pink1 ${ }^{+/-}$and $\mathrm{Pink1}^{-/-}$mice after virus challenge, as shown by decreased activity of both complex I (mean reduction, $16 \%$ and $50 \%$, respectively) and complex IV (mean reduction, $52 \%$ and $73 \%$, respectively) (Figure 10A). Mitochondrial mass, assessed as the mtDNA/gDNA ratio, was increased in $\mathrm{Pink1}^{+/-}$and $\mathrm{Pink1}^{-/-}$mice (Figure 10B), suggestive of defective turnover of damaged mitochondria. In accordance with our in vitro observations that PINK1 deficiency increased apoptosis in alveolar epithelial cells, in situ TUNEL assay of apoptotic responses showed increased positive signals in $\mathrm{Pink1}^{+/-}$and $\mathrm{Pink1}^{-/-}$ lungs (Figure 10, C and D), which suggests that PINK1 confers protection against lung injury. We confirmed the increased vulnerability to apoptosis in PINK1-deficient mice by assessing levels of the proapoptotic molecule BAX. Levels of BAX in Pink1 ${ }^{+/-}$and Pink1 $1^{-/}$lungs were enhanced after MHV68 infection (Figure 10, E and F, and Supplemental Figure 8A). We also analyzed the effects of MHV68 infection on OPA1, a mediator of mitochondrial fusion. OPA1 expression was particularly increased in infected $\mathrm{Pink1}^{-1-}$ mice (Figure 10, E and F, and Supplemental Figure 8A). MHV68 infection also caused induction of autophagy, as demonstrated by elevated LC3II in all mouse strains, but p62 accumulated only in the PINK1-deficient groups (Figure 10, E-G). 

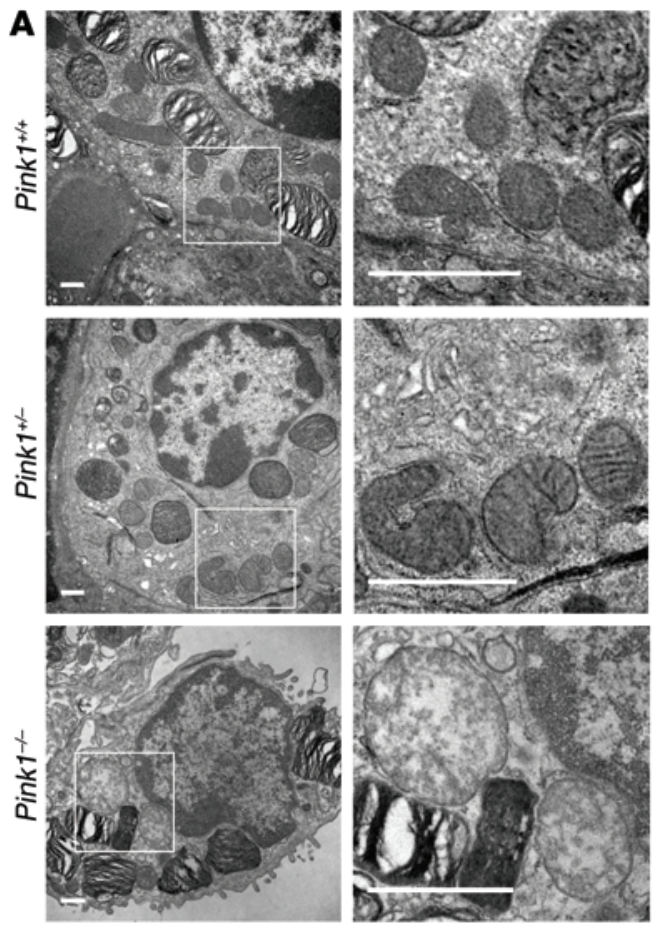

D

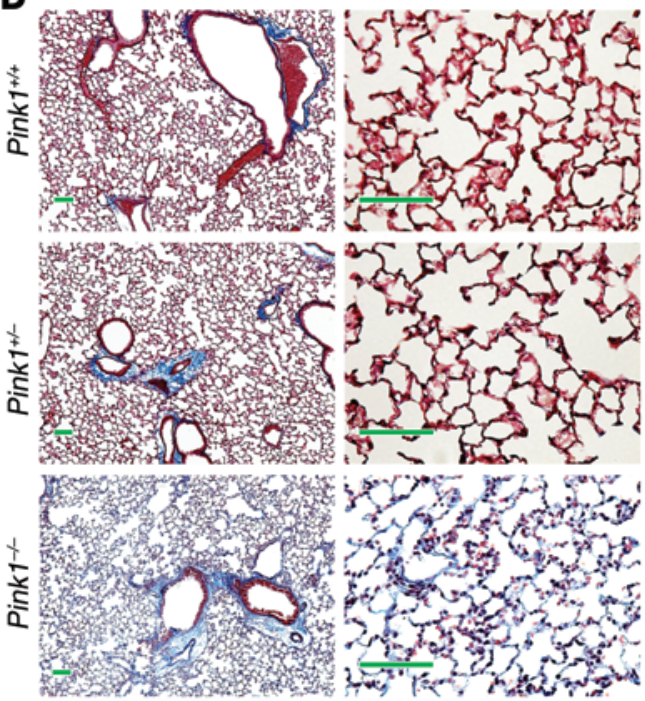

B
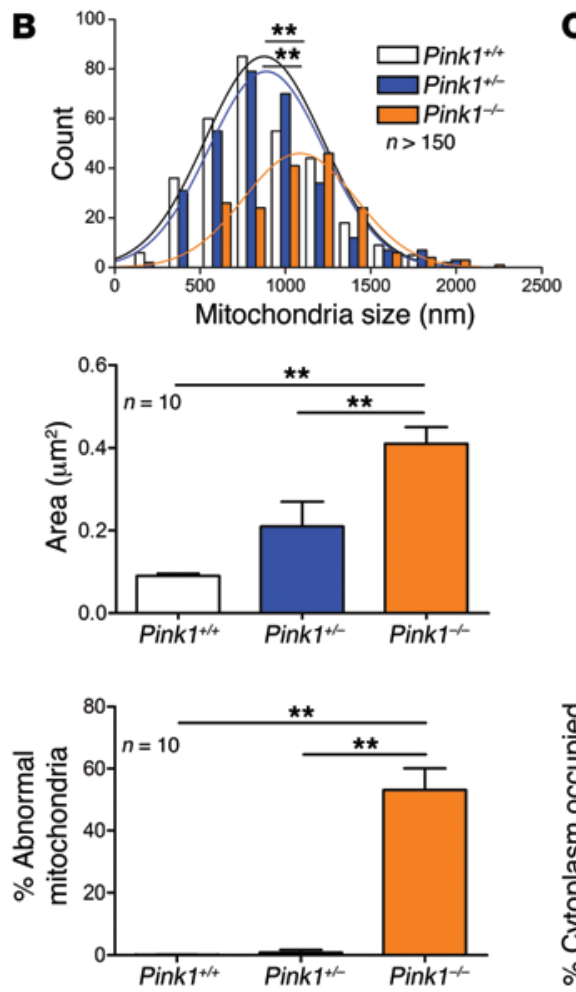

E
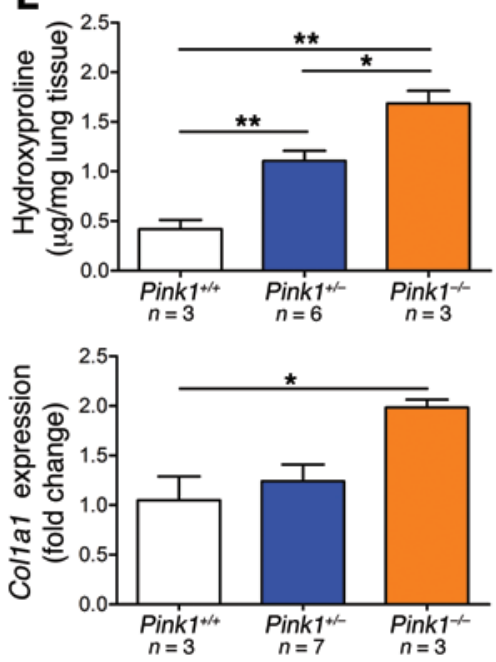
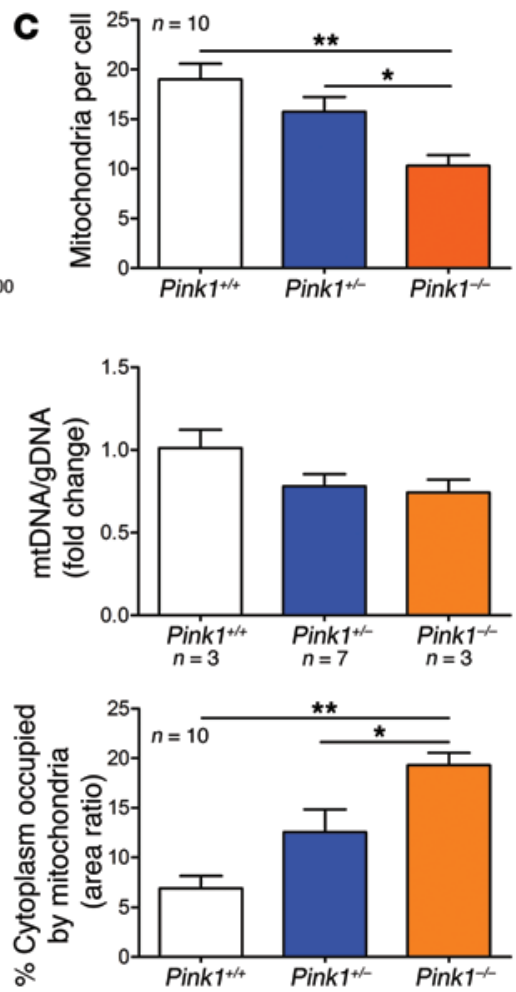

F
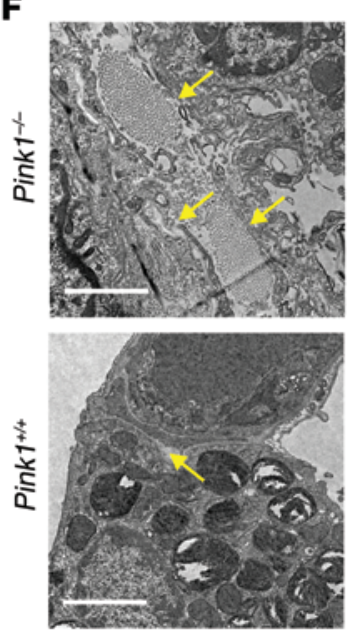

Figure 9. Altered mitochondrial quality control in AECIIs from PINK1-deficient mice. (A) Representative TEM ( $n=3$ per group) of AECIIs from Pink $7^{+/+}$ Pink $1^{+/}$, and Pink $1^{-/-}$mice. Mitochondrial profiles showed enlarged swollen mitochondria in Pink $1^{-/-}$AECIls. Boxed regions are shown enlarged at right. Scale bars: $500 \mathrm{~nm}$. (B) Frequency of mitochondria sizes, mitochondrial area, and percentage of abnormal mitochondria (swollen with evidence of severely disrupted cristae over all mitochondria) in Pink $1^{+/+}$, Pink $1^{+/}$, and Pink $1^{-/-}$AEClls (from TEM images). (C) Mitochondrial mass (number of mitochondria per cell and percentage of cytoplasm area occupied by mitochondria; assessed by quantitative morphometry of TEM images) and relative mtDNA/gDNA ratio. (D) Representative images of Masson trichrome staining in lung slides showing increased collagen deposition (blue) around airways in Pink $7^{+/-}$and Pink $1^{-/-}$ mice. Scale bars: $50 \mu \mathrm{m}$. (E) Significant increase in collagen deposition (assessed by hydroxyproline level) and Col1a1 expression in PINK1-deficient versus control mice. (F) Representative TEM of Pink $1^{+/+}$and Pink1 $1^{-/-}$mice. Collagen fibers in alveolar septa (arrows) surrounded AECIls in Pink $1^{-/-}$mice. Scale bars: $500 \mathrm{~nm}$. Data represent mean \pm SEM (C and E). ${ }^{*} P<0.05,{ }^{* *} P<0.01,1$-way ANOVA with post-hoc Bonferroni (B, C, and E).

We previously showed that MHV68 infection induces development of lung fibrosis in susceptible hosts $(11,35)$. Lung pathology of Pink1 $^{+/+}, \mathrm{Pink1}^{+/-}$, and Pink1 $1^{-/-}$mice was analyzed by Masson trichrome staining at day 15 after infection. Pink $1^{+/+}$mice developed only mild perivascular and peribronchial inflamma- tion after infection (Figure 11A). Pink1 $1^{+/-}$mice showed interstitial inflammation and fibrosis limited to the perivascular areas, but in stark contrast, Pink1 $^{-/-}$mice showed extended areas of interstitial fibrosis, which correlated with higher collagen I expression and collagen deposition measured by hydroxyproline levels (Fig- 

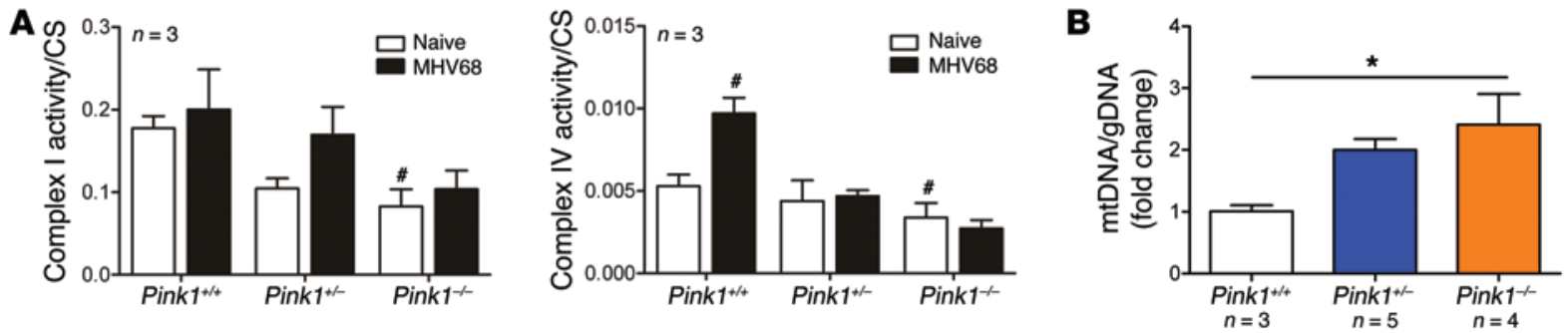

C
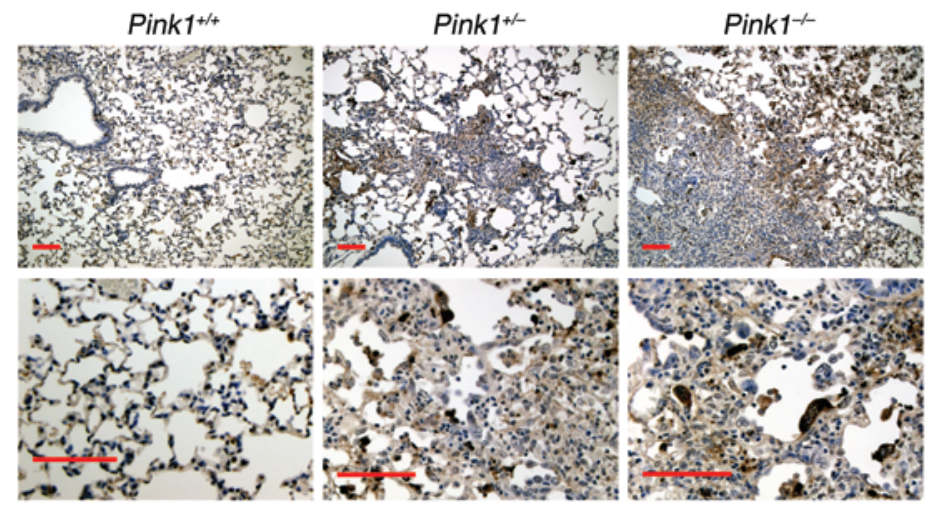

D

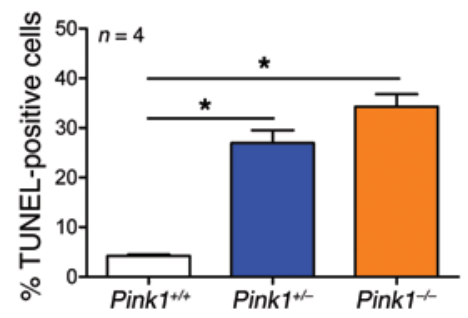

$\mathbf{E}$

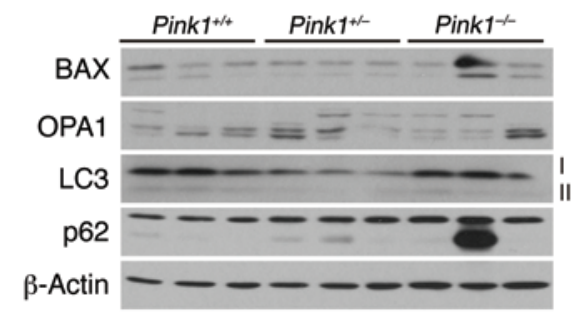

G

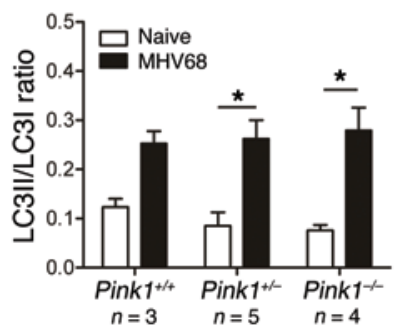

$\mathbf{F}$

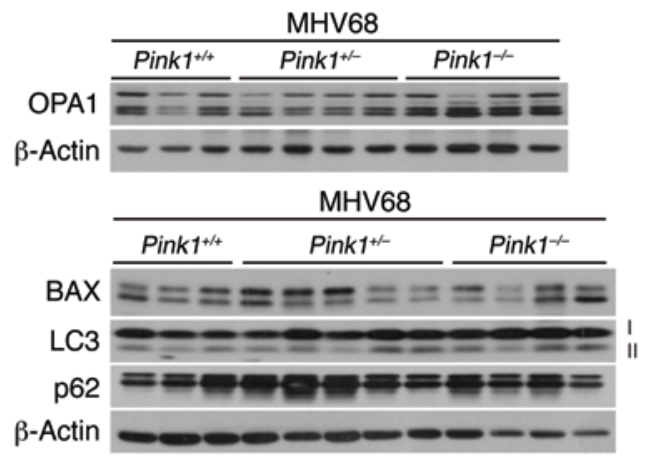

Figure 10. Mitochondrial dysfunction and increased cell apoptosis in PINIK1-deficient mice. (A) Complex I and complex IV activity, both basal and after MHV68 infection, was reduced in Pink1 $1^{-/-}$versus Pink1+/+ lung mitochondria. CS, citrate synthase. (B) Mitochondrial mass (assessed by mtDNA/gDNA ratio) in lungs of infected Pink $1^{+/+}$, Pink $1^{+/-}$, and Pink $1^{-/-}$mice. (C) Representative in situ TUNEL assay in lung sections at day 15 after MHV68 infection. Note the increase in positive signal (brown) in PINK1-deficient lungs. Scale bars: $50 \mu \mathrm{m}$. (D) Semiquantitative analyses showed significantly higher TUNEL-positive signal in PINK1-deficient versus control mice. (E and F) Immunoblot analyses in whole lung lysates from naive (E) and MHV68-infected (F) Pink1+/+, Pink $7^{+/-}$, and Pink $1^{-1-}$ mice for BAX, OPA1, and the autophagic markers LC3I/LC3II and p62. Blots were stripped and reblotted with $\beta$-actin for loading normalization. Each lane represents an individual mouse. (C) Density analyses of LC3 and p62. Data represent mean \pm SEM (A, B, D, and $\mathbf{C}){ }^{\#} P<0.05$ vs. Pink ${ }^{+/+}$; ${ }^{*} P<0.05$; 1 - (B and $\left.\mathbf{D}\right)$ or 2-way (A and $\left.\mathbf{G}\right)$ ANOVA with post-hoc Bonferroni.

ure 11, A-C). Additionally, we measured weight loss as a determinant of illness severity. PINK1-deficient mice had substantially more weight loss than controls; moreover, in contrast to the control group, this weight loss was not recovered spontaneously (Figure 11D). Control and PINK1-deficient mice showed similar viral loads, measured by number of copies of virus genome in the spleen (Orf50; Figure 11E), which indicated that fibrosis severity in PINK1-deficient mice was independent of viral persistence.
Moreover, PINK1 deficiency was accompanied by higher levels of the profibrotic mediators Tgfb and Fgf2 (Figure 11, F and G). Inflammatory responses were also more severe in PINK1-deficient mice than in control littermates, as determined by elevated mRNA levels of the proinflammatory cytokines Tnfa and Il $l 6$ and downmodulation of the antiinflammatory cytokine $I l 10$, despite BAL cell counts not differing significantly among the groups (Figure 11H and Supplemental Figure 8B). Quantitative analyses 
A
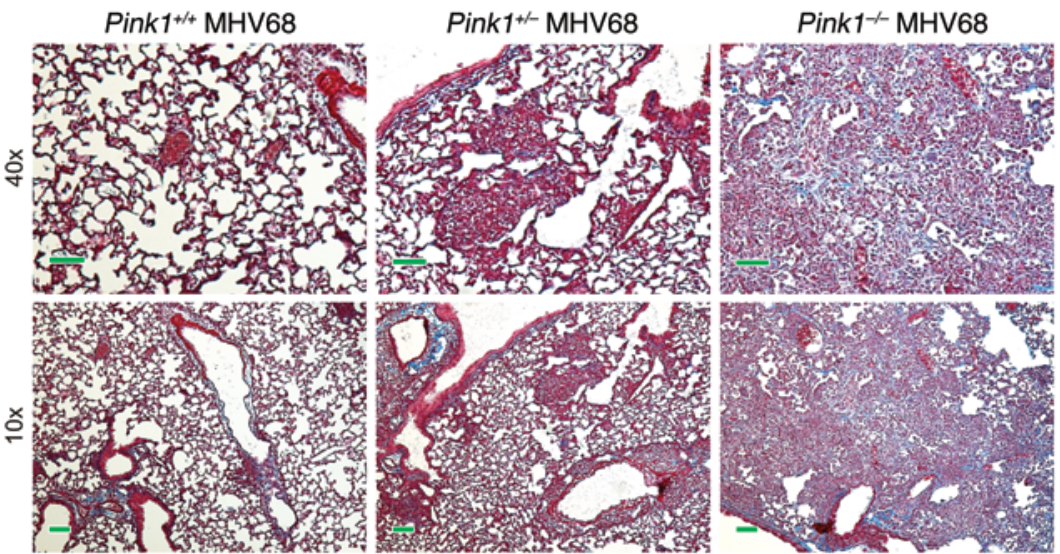

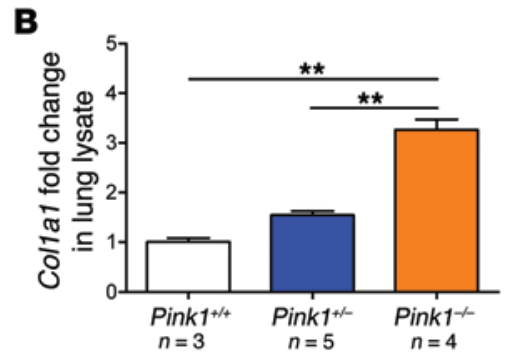

C

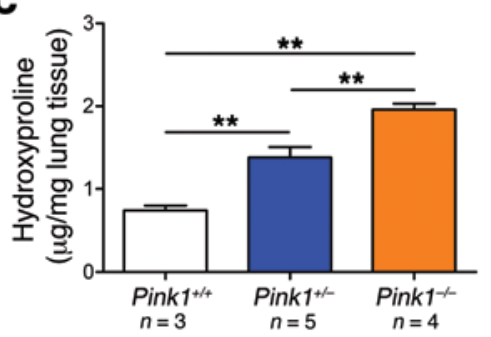

$\mathbf{F}$
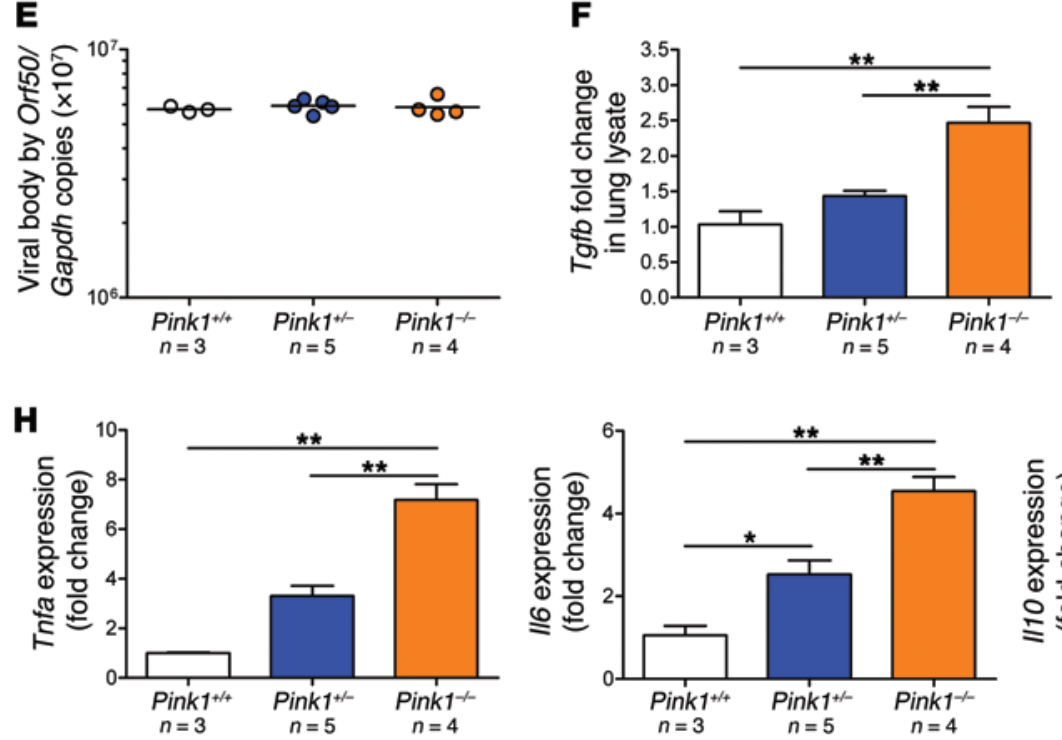

D

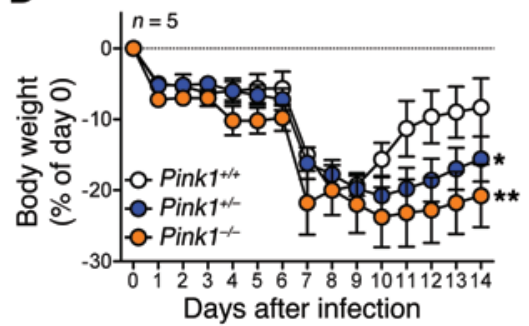

G

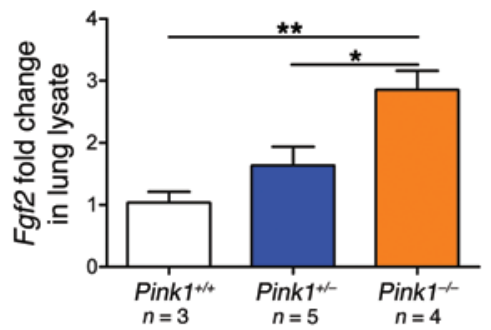

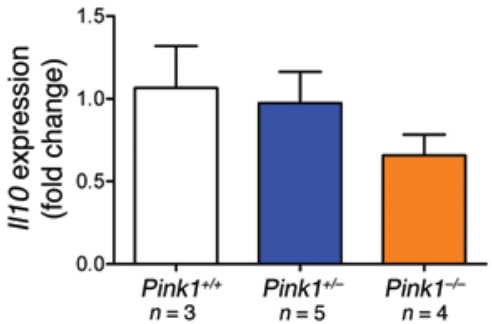

Figure 11. PINK1 deficiency increases susceptibility to lung fibrosis. (A) Representative Masson trichrome staining in Pink $1^{+/+}$, Pink $1^{+/-}$, and Pink $11^{-/-}$lung sections showed increased collagen deposition (blue) at day 15 after MHV68 infection. Scale bars: $10 \mu \mathrm{m}$ (40x); $50 \mu \mathrm{m}$ (10x). (B) Higher Col1a1 transcript levels in lungs of PINK1-deficient mice infected with MHV68 compared with control littermates. (C) Increased collagen deposition (assessed by hydroxyproline level) in lungs of PINK1-deficient mice after infection. (D) Weight loss after MHV68 infection was more severe in Pink1 $1^{-/-}$mice. (E) Viral load (assessed by qPCR) of individual mouse lungs 15 days after MHV68 infection. Bars represent geometric mean. (F and $\mathbf{G})$ Relative change in lung lysate Tgfb $(\mathbf{F})$ and Fgf2 (C) transcript levels after infection. (H) Relative change in Tnfa, II6, and I/10 mRNA levels after infection. Data represent mean \pm SEM (B-D and F-H). ${ }^{*} P<0.05,{ }^{* *} P<0.01$, 1-way ANOVA (B, C, and E-H) or 1-way repeated-measures ANOVA (D) with post-hoc Bonferroni.

of transcript levels of inducible nitric oxide synthase (Inos) and the like chitinase $Y m 1 / Y m 2$, as markers of classical and alternative macrophage activation, respectively, showed comparable expression between control and PINK1-deficient mice before and after MHV68 infection (Supplemental Figure 8C). Additionally, we isolated lung fibroblasts (passage 4) from Pink1+/+ and Pink1 ${ }^{-/}$mice and, using wound closing studies, found similar pro- liferation and migration properties in both groups (Supplemental Figure 8D). Since enhanced expression of NADPH oxidase 4 (NOX4) in fibroblasts has been associated with persistent fibrosis in aging mice (40), we also measured Nox4 transcript levels in isolated lung fibroblasts from Pink1 ${ }^{+/+}$and Pink1-/- mice. Comparable Nox4 expression was found in the presence and absence of TGF- $\beta$ stimulation in both mouse strains (Supplemental Figure 
$8 \mathrm{E})$, which suggested that there are not intrinsic differences in lung fibroblasts of PINK1-deficient mice.

To confirm the higher susceptibility to lung injury and fibrosis of PINK1-deficient mice, we used a second model of lung injury, the classic bleomycin-induced lung fibrosis model. Intratracheal instillation of bleomycin ( $2 \mathrm{U} / \mathrm{kg}$ weight) was performed in young (2-3 months) Pink1 ${ }^{+/+}$and Pink1 $1^{-/-}$mice. Similar to the observed response to MHV68 infection, Pink1 $1^{-/}$mice showed increased weight loss as well as stronger induction of inflammatory responses, as shown by significantly different BAL differential cell counts, upregulation of the proinflammatory cytokine Tnfa, and downmodulation of the antiinflammatory cytokine Il1O (Supplemental Figure 9, A-C). In addition, Pink1 $1^{-/-}$mice treated with bleomycin showed increased fibrosis severity and elevated collagen deposition, as assessed by hydroxyproline levels and collagen I expression (Supplemental Figure 9, D and E). Transcript levels of $\mathrm{Tg} f b$ were also elevated in $\mathrm{Pink1}^{-/-}$mice treated with bleomycin (Supplemental Figure 9F). Together, these data suggested that PINK1 has an important role maintaining mitochondrial homeostasis in AECIIs that might affect vulnerability to lung injury and development of lung fibrosis.

\section{Discussion}

Aging is a risk factor for many chronic disorders, including lung diseases. Observations in neurodegenerative and cardiovascular diseases suggest that a common denominator for age-related diseases is a decline in mitochondrial function. Here, we showed that age-related mitochondrial dysfunction and impaired mitophagy in the AECIIs may have a previously unrecognized role in the pathogenesis of IPF. We identified deficiency of PINK1 as a fundamental mechanism leading to accumulation of dysfunctional mitochondria in the AECIIs of IPF patient lungs. Moreover, we recapitulated the mitochondrial phenotype observed in the human IPF lung and the susceptibility to lung fibrosis in animal models of aging, ER stress, and PINK1 deficiency.

Similar to our findings in AECIIs, neurons with PINK1 mutations or PINK1 silencing show depolarized mitochondria, mitochondrial swelling, and enhanced susceptibility to apoptosis (41, 42). The susceptibility to PINK1 deficiency appears to relate to energy demands and cell type-specific expression. In concordance with our present results, PINK1 has been reported to be expressed higher in epithelial tissues and neural tissue and lower in tissues of mesenchymal origin, excepting muscle (43). Upregulation of PINK1 has been found in lung epithelial cells of patients with chronic obstructive pulmonary disease (44). Here, we found that AECIIs were highly susceptible to PINK1 deficiency, showing spontaneous mitochondrial dysfunction and predominance of enlarged and swollen mitochondria from a very early age. Interestingly, our present studies revealed that naive PINK1-deficient mice had increased profibrotic responses in the lung that resulted in mild collagen deposition in the absence of a second hit. AECIIs in the IPF lung have a chaotic hyperplasic and hypertrophic phenotype and also represent the primary source of mediators that promote fibroblast proliferation, fibroblast migration, angiogenesis, and production of extracellular matrix components (45). Accordingly, we showed here that PINK1-deficient AECIIs exposed to ER stress had increased expression of profibrotic factors. TGF- $\beta$ is a well- known profibrotic factor, and its expression was elevated in alveolar cells with deficient PINK1 expression. PINK1 deficiency was also associated with high levels of FGF2. In pulmonary fibrosis, FGF2 is expressed in epithelial cells and associated with fibroblast proliferation and angiogenesis (46). PINK1 deficiency has recently been shown to enhance release of inflammatory cytokines (including IL-1 $\beta$, TNF- $\alpha$, and IL-6) after injury in the brain, by a NF- $\mathrm{BB}^{-}$ dependent mechanism (47). Mitochondrial materials such as mtDNA that leak from damaged mitochondria have been reported to elucidate inflammatory responses $(48,49)$. Our present data also support a potential role for mtDNA to induce profibrotic responses, such as production of TGF- $\beta$ and FGF2, in lung cells.

PINK1 expression is induced by the canonical PTEN and its isoform PTENa (50). Fibroblasts and epithelial cells from IPF lungs have been found to express low levels of PTEN, with consequent persistent AKT activation. Mice with constitutive deficiency of PTEN develop spontaneous fibrosis by intrinsic defects in fibroblasts and negative regulation of their proliferative response $(51,52)$. Furthermore, conditional targeted deletion of PTEN in lung epithelium exacerbated injury and fibrosis after bleomycin treatment by an increase of active TGF- $\beta$ and loss of epithelial integrity (53). In support of a role for PTEN in PINK1 deficiency in IPF lungs, microarray analyses of the Lung Genomics Research Consortium (LGRC) cohort (http://www.lung-genomics.org/) showed that PINK1 expression correlated directly with expression levels of the gene set of the PTEN pathway (Supplemental Table 3). On the other hand, low PINK1 expression correlated with high expression of caspase and mitochondrial apoptotic signaling pathway gene sets (Supplemental Table 3), which supports our present findings of higher apoptotic responses in PINK1-deficient mouse lungs as well as the role of PTEN in lung epithelial integrity. Consistent with an upstream role of PTEN in regulation of PINK1 expression, PINK1-deficient mice did not exhibit alterations in expression of PTEN and phosphorylated AKT (data not shown).

Similar to our present findings, studies in heart and neurons suggest that there is an age-dependent decline in autophagic function that probably contributes to the accumulation of damaged mitochondria $(16,54)$. Defective autophagy has been observed in IPF-derived lung fibroblasts in response to TGF- $\beta$ and associated with myofibroblast transformation $(55,56)$. Additionally, previously documented accumulation of ubiquitinated molecules in AECIIs from IPF lungs could potentially be associated with impaired autophagy (56). Deficiency of PINK1 is associated with increased mitochondrial injury (42) and decreased ability to recruit parkin to depolarized mitochondria, both of which initiate mitophagy. Both these mechanisms may contribute to the accumulation of dysfunctional mitochondria observed herein. However, PINK1 may also serve to downregulate endogenous parkin (57), and there are other pathways to mitophagy (58). Regardless, there is consensus that mitochondria from PINK1-deficient cells are swollen and dysfunctional, with aberrant mitochondrial fission/fusion dynamics, and may be poorly engulfed into the autophagosome. An additional consideration is the previously reported alterations of lysosome degradation with age, which might contribute to the decline of mitochondrial turnover in the aging lung (59). Our in vitro findings with PINK1 expression knockdown demonstrated that mitochondrial mass increased 
significantly in PINK1-deficient cells in the presence of ER stress. Several studies have revealed structural and functional communications between mitochondria and the ER (60). During ER stress, physical coupling is accomplished by anchoring proteins such as MFN2, allowing increased $\mathrm{Ca}^{2+}$ transfer from ER to mitochondria, which enhances mitochondrial ATP production and bioenergetics. Thus, under persistent ER stress, mitochondria are exposed to a high $\mathrm{Ca}^{2+}$ concentration and might undergo massive swelling. Our present studies showed that ER stress modulated mitochondrial function in the AECIIs through downregulation of PINK1, increasing apoptotic mitochondrial responses. Because aging individuals are highly susceptible to ER stress, it is possible that age-related vulnerability to lung fibrosis might be associated with persistent ER stress and subsequent mitochondrial damage.

In summary, our present findings indicated that aging and ER stress have an important effect on the physiology of AECII mitochondria and influence susceptibility to lung fibrosis. We showed, for the first time to our knowledge, that aging and ER stress were associated with deficient expression of PINK1 in AECIIs. As observed in neurons, PINK1 regulated mitochondrial homeostasis in the AECIIs, and its reduction caused accumulation of enlarged damaged mitochondria, loss of cell viability, and activation of profibrotic responses. Additionally, we uncovered a role for mitochondrial material in the induction of cytokines with proliferative and profibrotic properties in the lung. These data suggest that therapeutic pathways based on induction of PINK1 and/or improvement of mitochondrial function, dynamics, and turnover might be useful in the treatment of fibrotic lung diseases.

\section{Methods}

\section{Animals and animal treatment}

Young (2-3 months) and old ( $\geq 18$ months) C57BL/6 and BALB/c mice (Jackson Laboratories and National Institute of Aging) were used to isolated primary AECIIs. PINK1-deficient mice were maintained as a heterozygous line and bred to generate $\mathrm{Pink1}^{+/+}, \mathrm{Pink1}^{+/}$, and Pink1 ${ }^{-1-}$ littermates (61). C57BL/6, Pink1 ${ }^{+/+}$, Pink1 ${ }^{+/}$, and Pink1 ${ }^{-/-}$mice were inoculated intranasally with saline solution or $5 \times 10^{4} \mathrm{PFU}$ MHV68 as described previously $(11,35,37,38)$. For some experiments, mice received an intratracheal dose of TM or vehicle control ( $2 \mu \mathrm{g} /$ mouse). TM was dissolved in DMSO $(5 \mathrm{mg} / \mathrm{ml})$, then diluted 1:1,000 in PBS for intratracheal injection. In some instances, mice were infected with MHV68 $\left(5 \times 10^{4} \mathrm{PFU}\right) 48$ hours after TM inoculation. Additionally, groups of young and old mice were inoculated intratracheally with TM (20 $\mu \mathrm{g} /$ mouse) plus chloroquine $(2 \mathrm{mg} / \mathrm{kg}$ weight). Mice were sacrificed, and organs harvested and fixed, 4 hours after treatment. For the bleomycin-induced fibrosis experiments, a dose of $2 \mathrm{U} / \mathrm{kg}$ (administered intratracheally) was used in 2- to 3-month-old Pink1 $1^{+/}$, Pink1 $1^{+/}$, and $P$ ink $1^{-/-}$mice. Mice were sacrificed for organ harvest at day 15 after instillation or infection.

\section{Isolation of type II cells and fibroblasts}

Human AECIIs were obtained from lung tissue as previously reported (8). Mouse AECIIs were obtained from young and old mice (2 mice per isolation). Briefly, lung tissue was digested with a mixture of dispase (Sigma-Aldrich), collagenase (Sigma-Aldrich), and DNAseI (SigmaAldrich), using a Gentle MACS (Miltenyi) dissociation instrument.
Cell suspensions were subjected to negative selection of $\mathrm{CD} 45^{+}$and CD16/32+ cells using MACS beads and columns (Miltenyi). AECIIs were identified using electron microscopy. Human and mouse fibroblasts were obtained from fresh lung tissue by trypsin dispersion and grown (passage 4-8) in Ham's F12 medium (Gibco) supplemented with $10 \%$ FBS (Gibco).

\section{Histopathology, immunofluorescence, and electron microscopy}

After sacrifice, lungs were perfused with $2 \%$ paraformaldehyde, followed by paraffin embedding or saturation in 30\% sucrose for 24 hours for frozen sections. Paraffin sections were stained with H\&E or Masson trichrome to determine changes in histopathology and fibrosis, respectively. Immunohistochemistry studies used anti-SP-C (Millipore-Chemicon, catalog no. 50-173-573); anti-TOM20 (Santa Cruz, catalog no. sc-11415), and anti-BiP (Cell Signaling, catalog no. 3177) antibodies. Immunofluorescence analyses were performed using antibodies against LC3 (Novus Biologicals, catalog no. NB100-2220), ATP synthase (Thermo Scientific, catalog no. MA1-930), SP-C (Millipore-Chemicon, catalog no. 50-173-573), p62 (Abcam, catalog no. ab56416), and LAMP1 (Abcam, catalog no. ab24170). Semiquantitative analyses of cells double-positive for SP-C and ATP synthase were performed in 5 nonoverlapping tissue fields per individual lung parenchyma evaluated under $\times 60$ magnification. Similarly, semiquantitative analyses for LC3 staining were performed in mouse lungs using 5 nonoverlapping tissue fields evaluated under $\times 40$ magnification. TUNEL assays were performed in paraffinized lung tissue sections using a dUTP HRP labeling kit (Chemicon) accordingly to the manufacturer's recommendations. Semiquantitative scoring of $\mathrm{TUNEL}^{+}$cells was performed on 5 nonoverlapping tissue fields per individual lung parenchyma evaluated under $\times 40$ magnification. The mean percentage of $\mathrm{TUNEL}^{+}$cells was calculated from all the fields from all animals.

For electron microscopy, tissue was fixed in 3\% glutaraldehyde and $2 \%$ paraformaldehyde in $0.1 \mathrm{M}$ sodium cacodylate buffer at $\mathrm{pH}$ 7.3. Morphometric analyses (number of mitochondria per cell and mitochondrial size) were performed using NIH ImageJ (version 1.43). At least 10 cells from low-magnification images $(\times 10,000)$ were used to count the number of mitochondria per AECII (identified by the presence of lamellar bodies). At least 100-150 individual mitochondria, from 3 different lungs per group at high magnification $(\times 25,000$ and $\times 50,000$ ), were used to assess perimeter and area.

\section{Cell lines, culture, and treatments}

Human lung adenocarcinoma cells (cell line A549) were cultured in DMEM (high glucose; Gibco) with 10\% FBS (Gibco), $50 \mathrm{U} / \mathrm{ml}$ penicillin, and $50 \mu \mathrm{g} / \mathrm{ml}$ streptomycin (Gibco) in $5 \% \mathrm{CO}_{2}$ at $37^{\circ} \mathrm{C}$. Serumstarved conditions were reached by reducing the FBS concentration to $0.1 \%$. A549 cells were treated with TM (1 or $10 \mu \mathrm{g} / \mathrm{ml}$; SigmaAldrich) alone for 24 hours, or in combination with bafilomycin A1 (10 nM; Sigma-Aldrich). For PINK1 overexpression, cells were transfected with PINK1 and 3× FLAG in pReceiver M14 (Genecopoeia) or empty pReceiver M14 (control); to knock down PINK1 expression, cells were transfected with PINK1 shRNA-encoding sequences (Origene) or control pRS shRNA vector. Treatments were performed beginning 24 hours after transfection for an additional 24 (FGF2 or IL-6 detection) or 6 (TGF- $\beta$ detection) hours.

A549 cells were treated with exogenous mtDNA ( $5 \mathrm{ng} / \mathrm{ml})$ for 24 hours. mtDNA was extracted from A549 mitochondria by differential 
centrifugation in STE buffer (250 mM sucrose, 10 mM Tris, 1 mM EGTA, $\mathrm{pH}$ 7.4; Sigma-Aldrich) at $4^{\circ} \mathrm{C}$, as previously described (62-64). Then mtDNA was purified and concentrated using DNeasy kits (Qiagen), according to the manufacturer's recommendations, along with genomic DNA from the nuclear fraction (as stimulation control).

Apoptosis was measured by flow cytometry using the annexin V$\mathrm{PE}$ apoptosis detection kit (BD), following the manufacturer's recommendations. Mitochondrial mass and depolarization were measured with MitoTracker Green FM and JC-1 probes (Molecular Probes), respectively. Cells were incubated for 30 minutes at $37^{\circ} \mathrm{C}$ with MitoTracker Green (100 nM) or JC-1 $(2 \mu \mathrm{M})$, trypsinized, and resuspended in PBS. Fluorescence intensity was measured by flow cytometry or in a plate reader (as recommended by the manufacturer).

\section{OCR}

OCR was measured using the Seahorse XF24 Extracellular Flux analyzer (Seahorse Bioscience). Following treatment with TM $(1 \mu \mathrm{g} / \mathrm{ml})$ for 1 hour, cells were rinsed with media, then resuspended in an unbuffered assay medium containing $8.3 \mathrm{~g} / \mathrm{l} \mathrm{DMEM}, 200 \mathrm{mmol} / \mathrm{lglu}$ tamax-1, $100 \mathrm{mmol} / \mathrm{l}$ sodium pyruvate, $25 \mathrm{mmol} / \mathrm{l}$ glucose, $1.85 \mathrm{~g} / \mathrm{l}$ sodium chloride, and phenol red. Cells were then spun into the wells of a collagen type I-coated Seahorse plate at $200 \mathrm{~g}$ for 5 minutes. After 28 minutes of equilibration, oligomycin $(20 \mu \mathrm{mol} / \mathrm{l})$ was injected into each well, followed by FCCP $(20 \mu \mathrm{mol} / \mathrm{l})$ at 45 minutes and rotenone $(20 \mu \mathrm{mol} / \mathrm{l})$ at 62 minutes.

\section{Mitochondrial complex I and IV activities}

Differential centrifugation in STE buffer $(250 \mathrm{mM}$ sucrose, $10 \mathrm{mM}$ Tris, $1 \mathrm{mM} \mathrm{EGTA,} \mathrm{pH} 7.4$ ) at $4^{\circ} \mathrm{C}$ was used to isolate lung mitochondria, as previously described (62-64). Complex I activity was determined in isolated mitochondria spectrophotometrically (at $340 \mathrm{~nm}$ ) monitoring NADH oxidation $(100 \mu \mathrm{M})$ in the presence of $10 \mu \mathrm{M}$ coenzyme Q1 with or without $25 \mu \mathrm{M}$ rotenone. Activity of complex IV (cytochrome c oxidase) was measured using reduced cytochrome $\mathrm{c}$ as substrate and monitoring the oxidation of cytochrome $\mathrm{c}$ at $550 \mathrm{~nm}$. Diluted supernatant containing $1-10 \mu \mathrm{g}$ protein was equilibrated to $30^{\circ} \mathrm{C}$ in $10 \mathrm{mM}$ potassium phosphate ( $\mathrm{pH}$ 7.0), and the reaction was initiated by addition of $50 \mu \mathrm{M}$ reduced cytochrome c.

\section{Western blot analyses}

Extracts from whole lung tissue samples as well as enriched mitochondrial and cytosolic fractions were prepared as previously described (65). The antibodies used were as follows: MFN1, TOM20, $\beta$-actin, DRP1, p-JNK, and BAX (all Santa Cruz); $\alpha$-tubulin, p-DRP1, JNK, AKT, and p-AKT (all Cell Signaling); OPA1, p62, and MFN2 (all Abcam); PINK1 (Novus); LC3 (MBL); PTEN (Millipore). Nitrocellulose membranes were used, except for LC3 and TOM20 detection (PVDF). After primary detection, membranes were stripped and reprobed for $\beta$-actin, $\alpha$-tubulin, or TOM20 as loading controls. Band intensities were quantified using NIH ImageJ (version 1.43).

\section{Quantitative PCR for gene expression, mtDNA/gDNA ratio, and viral load}

Copurification of RNA and DNA were performed in lung tissue, primary AECIIs, and cell lines using All Prep DNA/RNA kits (Qiagen), according to the manufacturer's recommendations. For gene expression, 1-step qRT-PCR was performed for the genes of interest and nor- malized using $18 S$ RNA as housekeeping gene. Premixed primers and probes were used for TGFB1, FGF2, PINK1, COL1A1, TNFA, IL6, Inos, and $Y m 1 / Y m 2$ (Integrated DNA Technologies).

Purification of DNA was performed in spleen samples using DNeasy kits (QIAGEN) to determine viral load. Quantitative PCR (qPCR) for MHV68 DNA was performed in spleen samples as described previously (38), using viral ORF50 primers (forward, GGCCGCAGACATTTAATGAC; reverse, GCCTCAACTTCTCTGGATATGCC) and host GAPDH as housekeeping gene (forward, CCTGCACCACCAACTGCTTAG; reverse, GTGGATGCAGGGATGATGTTC). To quantify the mtDNA/gDNA ratio, qPCR was used to amplify 1 gene of the mitochondrial genome ( $N d 2$ in mouse; mt16S in humans) and 1 gene of the nuclear genome (Gapdh in mouse; B2M in humans). Primer sequences were as follows: Gapdh forward, CCTGCACCACCAACTGCTTAG; Gapdh reverse, GTGGATGCAGGGATGATGTTC; $N d 2$ forward, CCCATTCCACTTCTGATTACC; $N d 2$ reverse, ATGATAGTAGAGTTGAGTAGCG; B2M forward, TGCTGTCTCCATGTTTGATGTATCT; B2M reverse, TCTCTGCTCCCCACCTCTAGGT; mt16S forward, GCCTTCCCCCGTAAATGATA, mt16S reverse, TTATGCGATTACCGGGCTCT.

\section{Hydroxyproline assay}

Frozen lung tissue samples were incubated in $250 \mu \mathrm{l}$ PBS, after which $250 \mu \mathrm{l}$ of $12 \mathrm{~N} \mathrm{HCl}$ was added, and samples were then hydrolyzed at $110^{\circ} \mathrm{C}$ overnight. Samples were cooled and neutralized with $10 \mathrm{~N}$ $\mathrm{NaOH} .100 \mu \mathrm{l}$ of each sample was mixed with $400 \mu \mathrm{l}$ oxidizing solution, consisting of $1.4 \%$ chloramine-T, $10 \%$ N-propanol, and $80 \%$ citrate-acetate buffer (5\% citric acid, 1.2\% glacial acetic acid, $7.25 \%$ sodium acetate, and $3.4 \%$ sodium hydroxide) in PBS. The mixture was incubated for 30 minutes at room temperature. Next, Ehrlich's solution (Sigma-Aldrich) was added, samples were incubated at $65^{\circ} \mathrm{C}$ for 30 minutes, and absorbance was measured at $570 \mathrm{~nm}$. A standard curve was generated using trans-4-hydroxy-L-proline (SigmaAldrich). Results were expressed as micrograms hydroxyproline per milligram lung tissue.

\section{Microarray experiments and data analysis}

Patient population. To evaluate the expression levels of candidate genes, we analyzed a total of 219 lung tissue samples collected by the LGRC, consisting of samples from IPF patients $(n=123)$ and normal histology samples from control subjects $(n=96)$. See Supplemental Table 1 for demographic and clinical characteristics of the study cohort.

Microarray assays. For microarray generation, labeling reactions were performed for each sample using Quick Amp Labeling Kit, 1 color (Agilent Technologies), and adding positive control 1 color RNA Spike-in Kit (Agilent Technologies). Using a starting concentration of 500 ng total RNA, an initial cDNA strand was synthesized using an oligo(dT) 24 primer containing a T7 RNA polymerase. The resulting cDNA was then used as a template to generate Cy3-labeled cRNA by a reverse transcriptase enzyme. After cRNA was obtained, a purification step was performed using RNeasy Mini Kit (Qiagen) followed by measurement of the yield and specific activity of each sample. In the last steps, cRNA was fragmented for 30 minutes in the dark at $60^{\circ} \mathrm{C}$, then hybridized to SurePrint G3 $8 \times 60$ (Agilent Technologies) for 17 hours at $65^{\circ} \mathrm{C}$. The array slides were washed with GE Wash Buffer 1 (Agilent Technologies) for 1 minute at room temperature, followed by GE Wash Buffer 2 (Agilent Technologies) 
for 1 minute at $37^{\circ} \mathrm{C}$; Triton $\mathrm{X}$ was added to wash buffers. Arrays were scanned using Microarray Scanner C (Agilent Technologies) following the manufacturer's protocol. For array readout, Feature Extraction software (version 10.7; Agilent Technologies) was used to normalize the processed signal, and cyclic-LOESS was performed using the bioconductor package. Complete datasets are available at the LGRC website (http://www.lung-genomics.org).

Microarray statistical analysis. To evaluate the differences in expression levels between candidate genes, we used the Mann-Whitney $U$ test for independent samples of the microarray ( $\log _{2}$-transformed expression values). A $P$ value less than 0.05 was considered statistically significant. To evaluate the correlation between gene sets and PINK1 expression in lung tissue microarrays, we used gene set analysis for quantitative data and the complete LGRC database.

Accession number. Complete datasets were deposited in GEO (accession no. GSE47460).

\section{Statistics}

Data were statistically analyzed using Prism 5 (Graph Pad). Groups' differences were calculated by 2-tailed Student's $t$ test or by 1- or 2-way ANOVA followed by post-hoc tests to determine pairs of groups with significant differences and family-wise error rate at $5 \%$. Results are presented as mean \pm SEM. A $P$ value less than 0.05 was considered significant.

\section{Study approval}

This study was approved by the IACUC of the University of Pittsburgh and adhered to NIH guidelines for the use of experimental animals. Human AECIIs and human lung fibroblasts were obtained from lung tissue collected from excess pathologic tissue after lung transplantation and organ donation, under protocols approved by the University of Pittsburgh IRB.

\section{Acknowledgments}

We thank M.T. Gladwin for insightful comments, M. Mosher and M. Ross for technical assistance with confocal images, J. Callio for PINK1-deficient mouse breeding; B.M. Juan-Guardela for gene preparations, T. Richards for statistical consulting, N. Kaminski for gene array analysis, and J. Pilewski for lung samples (supported by NIH grant P3O DK072506). The present study was supported by the Vascular Medicine Institute at the University of Pittsburgh, the Institute for Transfusion Medicine, and the Hemophilia Center of Western Pennsylvania (to A.L. Mora) and by NIH grants NS065789 and AG026389 (to C.T. Chu).

Address correspondence to: Ana L. Mora, Vascular Medicine Institute, Division of Pulmonary, Allergy and Critical Care Medicine, Department of Medicine, University of Pittsburgh, E-1246 BST, 200 Lothrop Street, Pittsburgh, Pennsylvania 15213, USA. Phone: 412.624.2291; E-mail: anamora@pitt.edu.
1. Selman M, Rojas M, Mora AL, Pardo A. Aging and interstitial lung diseases: unraveling an old forgotten player in the pathogenesis of lung fibrosis. Semin Respir Crit Care Med. 2010;31(5):607-617.

2. Raghu G, Weycker D, Edelsberg J, Bradford WZ, Oster G. Incidence and prevalence of idiopathic pulmonary fibrosis. Am J Respir Crit Care Med. 2006;174(7):810-816.

3. Castriotta RJ, et al. Workshop on idiopathic pulmonary fibrosis in older adults. Chest. 2010;138(3):693-703.

4. Ley B, Collard HR, King TE Jr. Clinical course and prediction of survival in idiopathic pulmonary fibrosis. Am J Respir Crit Care Med. 2011;183(4):431-440.

5. Nalysnyk L, Cid-Ruzafa J, Rotella P, Esser D. Incidence and prevalence of idiopathic pulmonary fibrosis: review of the literature. Eur Respir Rev. 2012;21(126):355-361.

6. Armanios MY, et al. Telomerase mutations in families with idiopathic pulmonary fibrosis. N Engl JMed. 2007;356(13):1317-1326.

7. Lawson WE, et al. Genetic mutations in surfactant protein $\mathrm{C}$ are a rare cause of sporadic cases of IPF. Thorax. 2004;59(11):977-980.

8. Korfei M, et al. Epithelial endoplasmic reticulum stress and apoptosis in sporadic idiopathic pulmonary fibrosis. Am J Respir Crit Care Med. 2008;178(8):838-846.

9. Lawson WE, et al. Endoplasmic reticulum stress enhances fibrotic remodeling in the lungs. Proc Natl Acad Sci US A. 2011;108(26):10562-10567.

10. Noble PW, Barkauskas CE, Jiang D. Pulmonary fibrosis: patterns and perpetrators. JClin Invest. 2012;122(8):2756-2762.

11. Torres-Gonzalez E, et al. Role of endoplasmic reticulum stress in age-related susceptibility to lung fibrosis. Am J Respir Cell Mol Biol.
2012;46(6):748-756.

12. Bratic A, Larsson NG. The role of mitochondria in aging. J Clin Invest. 2013;123(3):951-957.

13. Massaro GD, Gail DB, Massaro D. Lung oxygen consumption and mitochondria of alveolar epithelial and endothelial cells. J Appl Physiol. 1975;38(4):588-592.

14. Crapo JD, Peters-Golden M, Marsh-Salin J, Shelburne JS. Pathologic changes in the lungs of oxygen-adapted rats: a morphometric analysis. Lab Invest. 1978;39(6):640-653.

15. Vannuvel K, Renard P, Raes M, Arnould T. Functional and morphological impact of ER stress on mitochondria. J Cell Physiol. 2013;228(9):1802-1818.

16. Dutta D, Calvani R, Bernabei R, Leeuwenburgh C, Marzetti E. Contribution of impaired mitochondrial autophagy to cardiac aging: mechanisms and therapeutic opportunities. Circ Res. 2012;110(8):1125-1138.

17. Chu CT. A pivotal role for PINK1 and autophagy in mitochondrial quality control: implications for Parkinson disease. Hum Mol Genet. 2010;19(R1):R28-R37.

18. Narendra D, Walker JE, Youle R. Mitochondrial quality control mediated by PINK1 and Parkin: links to parkinsonism. Cold Spring Harb Perspect Biol. 2012;4(11):a011338.

19. Youle RJ, Narendra DP. Mechanisms of mitophagy. Nat Rev Mol Cell Biol. 2011;12(1):9-14.

20. Valente EM, et al. Hereditary early-onset Parkinson's disease caused by mutations in PINK1. Science. 2004;304(5674):1158-1160.

21. Scheele C, et al. Altered regulation of the PINK1 locus: a link between type 2 diabetes and neurodegeneration? FASEB J. 2007;21(13):3653-3665.

22. Anichtchik O, Diekmann H, Fleming A, Roach
A, Goldsmith P, Rubinsztein DC. Loss of PINK1 function affects development and results in neurodegeneration in zebrafish. J Neurosci. 2008;28(33):8199-8207.

23. Morais VA, et al. Parkinson's disease mutations in PINK1 result in decreased Complex I activity and deficient synaptic function. EMBO Mol Med. 2009;1(2):99-111.

24. Wang HL, et al. PARK6 PINK1 mutants are defective in maintaining mitochondrial membrane potential and inhibiting ROS formation of substantia nigra dopaminergic neurons. Biochim Biophys Acta. 2011;1812(6):674-684.

25. Liu W, Acin-Perez R, Geghman KD, Manfredi G, Lu B, Li C. Pink1 regulates the oxidative phosphorylation machinery via mitochondrial fission. Proc Natl Acad Sci U S A. 2011;108(31):12920-12924.

26. Unoki M, Nakamura Y. Growth-suppressive effects of BPOZ and EGR2, two genes involved in the PTEN signaling pathway. Oncogene. 2001;20(33):4457-4465.

27. Billia F, Hauck L, Konecny F, Rao V, Shen J, Mak TW. PTEN-inducible kinase 1 (PINK1)/Park6 is indispensable for normal heart function. Proc Natl Acad Sci U S A. 2011;108(23):9572-9577.

28. Ngoh GA, Papanicolaou KN, Walsh K. Loss of mitofusin 2 promotes endoplasmic reticulum stress. J Biol Chem. 2012;287(24):20321-20332.

29. Yuzefovych LV, Musiyenko SI, Wilson GL, Rachek LI. Mitochondrial DNA damage and dysfunction, and oxidative stress are associated with endoplasmic reticulum stress, protein degradation and apoptosis in high fat diet-induced insulin resistance mice. PLoS One. 2013;8(1):e54059.

30. Cali T, Ottolini D, Negro A, Brini M. Enhanced parkin levels favor ER-mitochondria crosstalk and guarantee $\mathrm{Ca}(2+)$ transfer to sustain 
cell bioenergetics. Biochim Biophys Acta. 2013;1832(4):495-508.

31. Lawson WE, et al. Endoplasmic reticulum stress in alveolar epithelial cells is prominent in IPF: association with altered surfactant protein processing and herpesvirus infection. Am J Physiol Lung Cell Mol Physiol. 2008;294(6):L1119-L1126.

32. Galluzzi L, Kepp O, Kroemer G. Mitochondria: master regulators of danger signalling. Nat Rev Mol Cell Biol. 2012;13(12):780-788.

33. Twig G, et al. Fission and selective fusion govern mitochondrial segregation and elimination by autophagy. EMBO J. 2008;27(2):433-446.

34. Gegg ME, Cooper JM, Schapira AH, Taanman JW. Silencing of PINK1 expression affects mitochondrial DNA and oxidative phosphorylation in dopaminergic cells. PLoS One. 2009;4(3):e4756.

35. Mora AL, et al. Lung infection with $\gamma$-herpesvirus induces progressive pulmonary fibrosis in Th2biased mice. Am J Physiol Lung Cell Mol Physiol. 2005;289(5):L711-L721.

36. Mora AL, et al. Activation of alveolar macrophages via the alternative pathway in herpesvirus-induced lung fibrosis. Am J Respir Cell Mol Biol. 2006;35(4):466-473.

37. Mora AL, et al. Control of virus reactivation arrests pulmonary herpesvirus-induced fibrosis in IFN-gamma receptor-deficient mice. Am J Respir Crit Care Med. 2007;175(11):1139-1150.

38. Krug LT, et al. Inhibition of NF-kappaB signaling reduces virus load and gammaherpesvirus-induced pulmonary fibrosis. Am J Pathol. 2010;177(2):608-621

39. Pozharskaya V, et al. Twist: a regulator of epithelial-mesenchymal transition in lung fibrosis. PLoS One. 2009;4(10):e7559.

40. Hecker L, et al. Reversal of persistent fibrosis in aging by targeting Nox4-Nrf2 redox imbalance. Sci Transl Med. 2014;6(231):231ra47.

41. Abramov AY, Gegg M, Grunewald A, Wood NW, Klein C, Schapira AH. Bioenergetic consequences of PINK1 mutations in Parkinson disease. PLoS One. 2011;6(10):e25622.

42. Dagda RK, et al. Loss of PINK1 function pro- motes mitophagy through effects on oxidative stress and mitochondrial fission. J Biol Chem. 2009;284(20):13843-13855.

43. Berthier A, et al. PINK1 displays tissue-specific subcellular location and regulates apoptosis and cell growth in breast cancer cells. Hum Pathol. 2011;42(1):75-87.

44. Mizumura K, et al. Mitophagy-dependent necroptosis contributes to the pathogenesis of COPD. J Clin Invest. 2014;124(9):3987-4003.

45. Selman M, Pardo A. Role of epithelial cells in idiopathic pulmonary fibrosis: from innocent targets to serial killers. Proc Am Thorac Soc. 2006;3(4):364-372.

46. Hanumegowda C, Farkas L, Kolb M. Angiogenesis in pulmonary fibrosis: too much or not enough? Chest. 2012;142(1):200-207.

47. Kim J, et al. PINK1 deficiency enhances inflammatory cytokine release from acutely prepared brain slices. Exp Neurobiol. 2013;22(1):38-44.

48. Zhang $\mathrm{Q}$, et al. Circulating mitochondrial DAMPs cause inflammatory responses to injury. Nature. 2010;464(7285):104-107.

49. Oka T, et al. Mitochondrial DNA that escapes from autophagy causes inflammation and heart failure. Nature. 2012;485(7397):251-255.

50. Liang $\mathrm{H}$, et al. PTEN $\alpha$, a PTEN isoform translated through alternative initiation, regulates mitochondrial function and energy metabolism. Cell Metab. 2014;19(5):836-848.

51. Xia H, et al. Pathological integrin signaling enhances proliferation of primary lung fibroblasts from patients with idiopathic pulmonary fibrosis. J Exp Med. 2008;205(7):1659-1672.

52. Xia H, Khalil W, Kahm J, Jessurun J, Kleidon J, Henke CA. Pathologic caveolin-1 regulation of PTEN in idiopathic pulmonary fibrosis. Am J Pathol. 2010;176(6):2626-2637.

53. Miyoshi K, et al. Epithelial Pten controls acute lung injury and fibrosis by regulating alveolar epithelial cell integrity. Am J Respir Crit Care Med. 2013;187(3):262-275.

54. Batlevi Y, La Spada AR. Mitochondrial autophagy in neural function, neurodegenerative disease, neuron cell death, and aging. Neurobiol Dis. 2011;43(1):46-51.

55. Patel AS, et al. Autophagy in idiopathic pulmonary fibrosis. PLoS One. 2012;7(7):e41394.

56. Araya J, et al. Insufficient autophagy in idiopathic pulmonary fibrosis. Am J Physiol Lung Cell Mol Physiol. 2013;304(1):L56-L69.

57. Rakovic A, et al. Effect of endogenous mutant and wild-type PINK1 on Parkin in fibroblasts from Parkinson disease patients. Hum Mol Genet. 2010;19(16):3124-3137.

58. Liu L, Sakakibara K, Chen Q, Okamoto K. Receptor-mediated mitophagy in yeast and mammalian systems. Cell Res. 2014;24(7):787-795.

59. Terman A, Gustafsson B, Brunk UT. The lysosomal-mitochondrial axis theory of postmitotic aging and cell death. Chem Biol Interact. 2006;163(1-2):29-37.

60. Malhotra JD, Kaufman RJ. ER stress and its functional link to mitochondria: role in cell survival and death. Cold Spring Harb Perspect Biol. 2011;3(9):a004424.

61. Dagda RK, et al. Mitochondrially localized PKA reverses mitochondrial pathology and dysfunction in a cellular model of Parkinson's disease. Cell Death Differ. 2011;18(12):1914-1923.

62. Shiva S, Brookes PS, Patel RP, Anderson PG, Darley-Usmar VM. Nitric oxide partitioning into mitochondrial membranes and the control of respiration at cytochrome coxidase. Proc Natl Acad Sci U S A. 2001;98(13):7212-7217.

63. Shiva $S$, et al. Nitrite augments tolerance to ischemia/reperfusion injury via the modulation of mitochondrial electron transfer. J Exp Med. 2007;204(9):2089-2102.

64. Mo L, et al. Nitrite activates AMP kinase to stimulate mitochondrial biogenesis independent of soluble guanylate cyclase. Free Radic Biol Med. 2012;53(7):1440-1450.

65. Velsor LW, Kariya C, Kachadourian R, Day BJ. Mitochondrial oxidative stress in the lungs of cystic fibrosis transmembrane conductance regulator protein mutant mice. Am J Respir Cell Mol Biol. 2006;35(5):579-586. 